\title{
48. COSTA RICA RIFT ZONE BASALTS: GEOCHEMICAL AND EXPERIMENTAL DATA FROM A POSSIBLE EXAMPLE OF MULTISTAGE MELTING'.
}

\author{
L. K. Autio and J. M. Rhodes, University of Massachusetts, Amherst, Massachusetts
}

\begin{abstract}
Chemical compositions and 1-atm. phase relations were determined for basalts drilled from Holes 501, 504A, 504B, 505, and 505B on Legs 68, 69, and 70 of the Deep Sea Drilling Project. Chemical, experimental, and petrographic data indicate that these basalts are moderately evolved $\left(\mathrm{Mg}^{\prime}\right.$ values from 0.60 to 0.70$)$, with olivine plus plagioclase and often clinopyroxene on the liquidus. Chemical stratigraphy was used to infer that sequential influxes of magma into a differentiating magma chamber or separate flows from different magma chambers or both had occurred.

Two major types of basalt were found to be interlayered: Group M, a rarely occurring type with major element chemistry and magmaphile element abundances within the range of the majority of ocean-floor basalts $\left(\mathrm{TiO}_{2} \cong 1.3 \%\right.$, $\mathrm{Na}_{2} \mathrm{O} \cong 2.5 \%, \mathrm{Zr} \cong 103 \mathrm{ppm}, \mathrm{Nb} \cong 2.5 \mathrm{ppm}$, and $\mathrm{Y} \cong 31 \mathrm{ppm}$ ); and Group D, a highly unusual series of basalt compositions that exhibit much lower magmaphile element abundances $\left(\mathrm{TiO}_{2}=0.75-1.2 \%, \mathrm{Na}_{2} \mathrm{O}=1.7-2.3 \%, \mathrm{Zr}=34-60\right.$ $\mathrm{ppm}, \mathrm{Nb}=0.5-1.2 \mathrm{ppm}$, and $\mathrm{Y}=16-27 \mathrm{ppm})$. The liquidus temperatures of the Group $\mathrm{D}$ basalts are high (1230$1260^{\circ} \mathrm{C}$ ) compared with those of other ocean-floor basalts of similar $\mathrm{Mg}^{\prime}$ values. They have high $\mathrm{CaO} / \mathrm{Na}_{2} \mathrm{O}$ ratios (5-8) and are calculated to be in equilibrium with unusually calcic plagioclase $\left(\mathrm{An}_{78-84}\right)$.

The two basalt groups cannot be related by fractionation processes. However, constant $\mathrm{Zr} / \mathrm{Nb}$ ratios $(\geq 40)$ for the two groups suggest a single mantle source, with differences in magmaphile element abundances and other element ratios (e.g., $\mathrm{Zr} / \mathrm{Ti}, \mathrm{Zr} / \mathrm{Y}, \mathrm{Ce} / \mathrm{Yb}$ ) arising through sequential melting of the same source. Magmas similar to Group $\mathrm{D}$, if mixed with more typical mid-ocean-ridge basalt (MORB) magmas in shallow magma chambers, could provide a source for the highly calcic plagioclase phenocrysts that appear in more common (i.e., less depleted) phyric ocean-floor basalts.
\end{abstract}

\section{INTRODUCTION}

Our purpose in making this study was to examine the chemistry of rock samples from the Costa Rica Rift zone and relate it to the petrographic and lithologic units described by Etoubleau et al. (this volume). Chemical and 1-atm. experimental melting studies were combined to provide information on the processes responsible for the generation and evolution of the Costa Rica Rift zone basaltic magmas.

Basalt chemistry and phase relations were examined for 18 samples from Hole 501, 1 sample from Hole 504A, 65 samples from Hole 504B, 1 sample from Hole 505A, and 4 samples from Hole 505B. These holes lie south of the modern Costa Rica Rift, from which the basalt is presumed to be derived, in crust 5.9 m.y. old (Holes $501,504 \mathrm{~A}$, and 504B) or $3.9 \mathrm{~m} . \mathrm{y}$. old (Holes 505A and 505B) (see site chapters, this volume). The basalts from all the locations are chemically similar. This paper concentrates on the samples from Holes 504B and 501. Basement penetration and recovery data for these sites are given in the site chapters.

In terms of chemistry, all the basalts are olivine (0$5 \%)$ to quartz $(0-2 \%)$ normative tholeiites. The samples we studied were aphyric to sparsely phyric $(0-10 \%$ phenocrysts) and approximated the liquid composition, with plagioclase as the most common phenocryst phase and smaller amounts of clinopyroxene, olivine, and spinel. Relatively minor hydrothermal alteration was manifested as small veins.

\footnotetext{
${ }^{1}$ Cann, J. R., Langseth, M. G., Honnorez, J., Von Herzen, R. P., White, S. M., et al., Init. Repts. DSDP, 69: Washington (U.S. Govt, Printing Office).
}

The major and trace element chemistry for all the samples is shown in Tables 1 to 3 , with rare earth element (REE) abundances for three selected samples given in Table 4. Samples are listed in order of increasing depth. Also indicated in Tables 1 to 3 are basalt types, which are based on the chemistry of the basalts according to X-ray fluorescence (XRF) analyses. The $\mathrm{Mg}^{\prime}$ values listed in these tables refer to molar $\mathrm{MgO} /(\mathrm{MgO}+$ $\mathrm{FeO})$ where $\mathrm{Fe}^{3+} /\left(\mathrm{Fe}^{2+}+\mathrm{Fe}^{3+}\right)$ is defined as 0.1 . The same convention for $\mathrm{Fe}^{3+}$ allocation has been used in norm calculations after Walker et al. (1979), which are shown in Figures 1 and 2.

Table 5 lists the average chemical composition of the basalts of the types found in Hole 504B (this study). Notations and calculations are the same in this table as in Tables 1 to 3. Preliminary results of the 1-atm. melting studies performed on seven samples of the basalt are given in Table 6.

\section{ANALYTICAL AND EXPERIMENTAL TECHNIQUES}

Before analysis, core samples (10-20 g) were crushed in a tungsten carbide shatterbox. All samples were analyzed on an automated Siemens SRS-2 X-ray fluorescence spectrometer. Powders for major element XRF analysis were ignited for $1 \mathrm{hr}$. at $1000^{\circ} \mathrm{C}$ in air to dry and oxidize them. All major elements except $\mathrm{Na}$ were analyzed on lanthanum-bearing lithium borate fused glass disks (Norrish and Hutton, 1969) using a chromium tube. Sodium was measured independently on an IL 443 flame photometer that employed a lithium internal standard; USGS standards AGV-1 and PCC-1 were used as calibration standards. Unfired 50-mg aliquots of powder were digested in hydrogen fluoride for 80 minutes at $110^{\circ} \mathrm{C}$ in sealed Nalgene polysulfone bottles and then made up to volume with boric acid solution (Bernas, 1968). The analytical precision for $\mathrm{Na}$ is 5 to $10 \%$.

Trace elements were determined by XRF analysis (Y, Sr, Rb, and $\mathrm{Ga}$ with a molybdenum tube; $\mathrm{Zr}, \mathrm{Nb}, \mathrm{Cr}, \mathrm{V}$, and $\mathrm{Ni}$ with a gold tube) on unfired pressed powder pellets. Corrections were made for back- 


\section{K. AUTIO, J. M. RHODES}

Table 1. Chemical composition of basalts from Hole 504B.

\begin{tabular}{|c|c|c|c|c|c|c|c|c|c|c|c|c|c|c|c|c|c|}
\hline Sample & 504B-1 & $504 \mathrm{~B}-2$ & 504B-3 & $504 \mathrm{~B}-4$ & $504 \mathrm{~B}-5$ & $504 \mathrm{~B}-6$ & $504 \mathrm{~B}-7$ & $504 \mathrm{~B}-8$ & 504B-9 & $504 \mathrm{~B}-10$ & $504 \mathrm{~B}-11$ & $504 \mathrm{~B}-12$ & $504 \mathrm{~B}-13$ & 504B-14 & $504 \mathrm{~B}-15$ & $504 \mathrm{~B}-16$ & 504B-17 \\
\hline Core-Section ${ }^{a}$ & 3-1 (44) & $4-4(10)$ & 5-2 (37) & $6-1(60)$ & 6-2 (20) & $6-2(140)$ & 7-1 (16) & $8-2(24)$ & $8-5(72)$ & $9-2(62)$ & $10-2(50)$ & $11-2(105)$ & $12-1(84)$ & $13-3$ (107) & $14-1(129)$ & $15-1(60)$ & $15-3(89)$ \\
\hline Depth $(m)^{b}$ & 3751.9 & 3758.1 & 3764.9 & 3772.6 & 3773.7 & 3774.9 & 3781.2 & 3791.7 & 3796.7 & 3801.2 & 3810.0 & 3819.6 & $3826.9^{\prime}$ & 3839.1 & 3845.3 & 3849.1 & 3852.4 \\
\hline Basalt Type ${ }^{\mathrm{c}}$ & D1 & D4 & D4 & D4 & D4 & D4 & D4 & D4 & D4 & D4 & D3 & DI & D4 & D4 & D4 & D4 & D4 \\
\hline \multicolumn{18}{|c|}{ Major elements (wt.\%) } \\
\hline $\mathrm{SiO}_{2}$ & 50.6 & 50.3 & 50.1 & 48.9 & 49.5 & 49.9 & 49.5 & 50.6 & 49.8 & 50.2 & 50.1 & 50.6 & 50.8 & 50.6 & 50.4 & 50.5 & 50.7 \\
\hline $\mathrm{TiO}_{2}$ & 1.09 & 0.90 & 0.90 & 0.91 & 0.94 & 0.92 & 0.91 & 0.90 & 0.88 & 0.93 & 1.00 & 1.12 & 0.96 & 0.95 & 0.94 & 0.96 & 0.92 \\
\hline $\mathrm{Al}_{2} \mathrm{O}_{3}$ & 15.3 & 16.5 & 16.4 & 16.0 & 16.2 & 16.7 & 16.3 & 16.5 & 16.0 & 15.9 & 15.6 & 17.7 & 15.6 & 15.6 & 15.6 & 15.4 & 15.4 \\
\hline $\mathrm{Fe}_{2} \mathrm{O}_{3} \cdot \mathrm{d}$ & 11.30 & 9.54 & 9.78 & 10.12 & 9.53 & 9.28 & 9.96 & 8.83 & 9.41 & 9.95 & 10.32 & 9.84 & 9.73 & 9.86 & 10.16 & 9.90 & 10.54 \\
\hline $\mathrm{MnO}$ & 0.18 & 0.18 & 0.19 & 0.21 & 0.17 & 0.17 & 0.18 & 0.16 & 0.17 & 0.18 & 0.17 & 0.13 & 0.18 & 0.16 & 0.19 & 0.18 & 0.17 \\
\hline $\mathrm{MgO}$ & 7.85 & 7.25 & 7.81 & 8.45 & 8.75 & 8.14 & 7.53 & 8.18 & 8.31 & 7.95 & 7.99 & 7.47 & 8.28 & 8.13 & 8.51 & 8.28 & 8.09 \\
\hline $\mathrm{CaO}$ & 12.06 & 13.26 & 13.22 & 12.38 & 12.57 & 12.40 & 13.16 & 12.98 & 12.95 & 12.60 & 12.17 & 10.60 & 12.62 & 12.80 & 12.76 & 12.79 & 12.61 \\
\hline $\mathrm{Na}_{2} \mathrm{O}$ & 2.1 & 1.9 & 1.9 & 2.0 & 2.0 & 1.9 & 1.9 & 2.2 & 1.9 & 2.0 & 2.1 & 2.3 & 2.1 & 2.2 & 1.8 & 1.9 & 2.0 \\
\hline $\mathrm{K}_{2} \mathrm{O}$ & 0.27 & 0.09 & 0.11 & 0.12 & 0.11 & 0.16 & 0.18 & 0.05 & 0.04 & 0.21 & 0.25 & 0.46 & 0.18 & 0.14 & 0.07 & 0.14 & 0.36 \\
\hline $\mathrm{P}_{2} \mathrm{O}_{5}$ & 0.12 & 0.07 & 0.08 & 0.10 & 0.09 & 0.09 & 0.09 & 0.07 & 0.06 & 0.09 & 0.09 & 0.10 & 0.08 & 0.10 & 0.07 & 0.08 & 0.07 \\
\hline Total & 100.83 & 99.99 & 100.49 & 99.15 & 99.86 & 99.66 & 99.71 & 100.47 & 99.52 & 100.01 & 99.79 & 100.32 & 100.53 & 100.54 & 100.50 & 100.13 & 100.86 \\
\hline \multicolumn{18}{|c|}{ Trace elements (ppm) } \\
\hline $\mathrm{Rb}$ & 4.8 & 1.3 & 1.8 & 1.5 & 1.5 & 1.4 & 1.7 & 0.6 & $<0.5$ & 4.0 & 4.8 & 7.9 & 3.4 & 1.6 & $<0.5$ & 1.9 & 7.3 \\
\hline $\mathrm{Sr}$ & 71 & 68 & 71 & 68 & 75 & 71 & 70 & 68 & 65 & 57 & 78 & 94 & 75 & 76 & 63 & 63 & 61 \\
\hline Y & 16.4 & 21.1 & 23.1 & 22.7 & 23.4 & 21.6 & 22.0 & 21.5 & 21.7 & 24.6 & 24.0 & 26.3 & 24.3 & 22.8 & 23.9 & 25.3 & 22.7 \\
\hline Ga & 16.7 & 14.9 & 16.1 & 15.1 & 16.0 & 16.0 & 14.9 & 15.4 & 15.7 & 16.0 & 15.2 & 16.4 & 15.4 & 16.5 & 15.2 & 16.8 & 16.2 \\
\hline $\mathrm{Zr}$ & 54 & 46 & 46 & 45 & 53 & 47 & 45 & 45 & 43 & 43 & 51 & 54 & 50 & 49 & 46 & 48 & 44 \\
\hline $\mathrm{Nb}$ & $<0.5$ & $<0.5$ & 0.6 & 0.7 & 1.1 & $<0.5$ & 0.5 & $<0.5$ & 0.7 & $<0.5$ & 0.6 & 0.5 & 0.8 & $<0.5$ & $<0.5$ & 0.7 & 0.5 \\
\hline $\mathrm{Zn}$ & 90 & 75 & 75 & 81 & 75 & 77 & 74 & 67 & 66 & 87 & 74 & 82 & 77 & 75 & 84 & 90 & 78 \\
\hline $\mathrm{Ni}$ & 77 & 131 & 142 & 145 & 153 & 144 & 133 & 125 & 120 & 109 & 94 & 78 & 99 & 81 & 110 & 115 & 76 \\
\hline $\mathrm{Cr}$ & 209 & 437 & 440 & 480 & 386 & 463 & 425 & 395 & 385 & 294 & 377 & 423 & 374 & 356 & 369 & 364 & 282 \\
\hline v & 327 & 248 & 254 & 273 & 241 & 260 & $24 !$ & 262 & 231 & 304 & 268 & 298 & 273 & 260 & 288 & 347 & 278 \\
\hline $\mathrm{Mg}^{\prime}$ value ${ }^{\mathrm{e}}$ & 0.605 & 0.626 & 0.637 & 0.648 & 0.669 & 0.659 & 0.625 & 0.671 & 0.660 & 0.637 & 0.630 & 0.626 & 0.652 & 0.645 & 0.648 & 0.648 & 0.628 \\
\hline $\mathrm{FeO}$ ed & 10.17 & 8.58 & 8.80 & 9.11 & 8.58 & 8.35 & 8.96 & 7.95 & 8.47 & 8.95 & 9.29 & 8.85 & 8.76 & 8.87 & 9.14 & 8.91 & 9.48 \\
\hline $\mathrm{CaO} / \mathrm{Na}_{2} \mathrm{O}$ & 5.74 & 6.98 & 6.96 & 6.19 & 6.29 & 6.53 & 7.74 & 5.90 & 6.82 & 6.30 & 5.80 & 4.61 & 6.01 & 5.82 & 7.09 & 6.73 & 6.31 \\
\hline
\end{tabular}

a Level (in parentheses) in $\mathrm{cm}$.

Below rig floor.

Basalt type refers to chemical units discussed in the text.

$\mathrm{Fe}_{2} \mathrm{O}_{3}{ }^{\circ}$ and $\mathrm{FeO} \mathrm{O}^{*}$ refer to total $\mathrm{Fe}$ expressed as $\mathrm{Fe}_{2} \mathrm{O}_{3}$ or $\mathrm{FeO}$.

' Calculation of $\mathrm{Mg}^{\prime}$ ' value is discussed in the text.

Table 1. (Continued).

\begin{tabular}{|c|c|c|c|c|c|c|c|c|c|c|c|c|c|c|c|}
\hline Sample & 504B-35 & 504B-36 & 504B-37 & $504 \mathrm{~B}-38$ & 504B-39 & $504 \mathrm{~B}-40$ & $504 \mathrm{~B}-41$ & $504 B-42$ & $504 \mathrm{~B}-43$ & $504 \mathrm{~B}-44$ & $504 \mathrm{~B}-45$ & $504 \mathrm{~B}-46$ & $504 \mathrm{~B}-47$ & $504 B-48$ & $504 \mathrm{~B}-49$ \\
\hline Core-Section ${ }^{a}$ & $40-2(87)$ & $40-5(1)$ & $41-2(70)$ & $42-1(31)$ & 43-1 (16) & $44-2$ (1) & $45-2(1)$ & $46-1$ (108) & $46-3(100)$ & $47-2(50)$ & $48-3(79)$ & $49-1$ (139) & $51-1(78)$ & $52-1(100)$ & $52-3(52)$ \\
\hline Depth $(m)^{b}$ & 4055.4 & 4057.0 & 4059.7 & 4066.3 & 4075.7 & 4086.0 & 4095.0 & 4103.1 & 4106.5 & 4113.5 & 4124.3 & 4130.9 & 4143.8 & 4153.0 & 4155.5 \\
\hline Basalt Type ${ }^{\mathrm{c}}$ & D7 & D7 & D7 & D4 & D4 & D4 & D4 & D4 & D4 & D4 & D6 & D6 & D6 & D6 & D6 \\
\hline \multicolumn{16}{|c|}{ Major elements (wt. $\sigma_{0}$ ) } \\
\hline $\mathrm{SiO}_{2}$ & 49.8 & 50.3 & 50.2 & 50.8 & 51.1 & 50.2 & 50.1 & 50.9 & 50.5 & 50.1 & 51.6 & 50.5 & 50.1 & 51.0 & 50.4 \\
\hline $\mathrm{TiO}_{2}$ & 0.77 & 0.76 & 0.78 & 0.95 & 0.90 & 0.86 & 0.87 & 0.93 & 0.94 & 0.92 & 0.79 & 0.84 & 0.81 & 0.81 & 0.86 \\
\hline $\mathrm{Al}_{2} \mathrm{O}_{3}$ & 16.4 & 16.8 & 16.3 & 16.2 & 15.6 & 16.1 & 16.0 & 15.4 & 14.8 & 15.0 & 15.9 & 16.1 & 16.4 & 16.8 & 16.2 \\
\hline $\mathrm{Fe}_{2} \mathrm{O}_{3} \cdot \mathrm{d}$ & 9.00 & 8.94 & 9.25 & 9.30 & 9.50 & 9.82 & 9.86 & 10.19 & 10.27 & 11.44 & 8.46 & 9.64 & 9.12 & 8.80 & 9.37 \\
\hline Mno & 0.14 & 0.13 & 0.14 & 0.14 & 0.16 & 0.14 & 0.14 & 0.16 & 0.18 & 0.12 & 0.13 & 0.16 & 0.13 & 0.14 & 0.14 \\
\hline $\mathrm{MgO}$ & 8.85 & 8.87 & 9.44 & 8.22 & 8.38 & 7.53 & 7.70 & 8.26 & 8.49 & 9.65 & 8.12 & 8.10 & 8.40 & 8.31 & 8.45 \\
\hline $\mathrm{CaO}$ & 12.93 & 12.95 & 12.60 & 12.93 & 12.57 & 13.39 & 13.01 & 12.72 & 12.84 & 10.92 & 12.97 & 12.82 & 13.01 & 13.30 & 13.02 \\
\hline $\mathrm{Na}_{2} \mathrm{O}$ & 1.8 & 1.8 & 1.9 & 2.2 & 2.1 & 2.0 & 2.1 & 2.0 & 2.0 & 1.8 & 2.0 & 1.7 & 1.9 & 2.1 & 1.9 \\
\hline $\mathrm{K}_{2} \mathrm{O}$ & 0.06 & 0.07 & 0.05 & 0.08 & 0.09 & 0.05 & 0.06 & 0.12 & 0.06 & 0.20 & 0.09 & 0.09 & 0.04 & 0.05 & 0.04 \\
\hline $\mathrm{P}_{2} \mathrm{O}_{5}$ & 0.05 & 0.04 & 0.03 & 0.06 & 0.06 & 0.05 & 0.03 & 0.06 & 0.06 & 0.05 & 0.03 & 0.04 & 0.07 & 0.05 & 0.05 \\
\hline Total & 99.80 & 100.66 & 100.69 & 100.88 & 100.46 & 100.14 & 99.87 & 100.74 & 100.14 & 100.20 & 100.09 & 99.99 & 99.98 & 101.36 & 100.43 \\
\hline \multicolumn{16}{|c|}{ Trace elements (ppm) } \\
\hline $\mathrm{Rb}$ & 0.5 & 0.9 & 0.8 & $<0.5$ & $<0.5$ & $<0.5$ & 0.7 & 1.5 & $<0.5$ & 2.6 & $<0.5$ & 0.5 & $<0.5$ & $<0.5$ & $<0.5$ \\
\hline $\mathrm{Sr}$ & 61 & 60 & 60 & 59 & 59 & 58 & 58 & 62 & 59 & 54 & 47 & 50 & 62 & 63 & 62 \\
\hline Y & 20.7 & 19.9 & 20.1 & 25.2 & 24.5 & 23.5 & 24.1 & 24.2 & 25.5 & 22.6 & 21.6 & 23.1 & 20.8 & 21.0 & 21.9 \\
\hline $\mathrm{Ga}$ & 15.2 & 14.5 & 14.4 & 15.6 & 15.9 & 15.0 & 16.3 & 15.8 & 14.3 & 12.8 & 14.7 & 14.9 & 16.0 & 15.6 & 16.4 \\
\hline $\mathrm{Zr}$ & 40 & 39 & 40 & 48 & 45 & 42 & 45 & 48 & 53 & 45 & 38 & 41 & 39 & 40 & 42 \\
\hline $\mathrm{Nb}$ & 0.7 & 1.0 & 1.0 & 0.5 & $<0.5$ & 0.8 & $<0.5$ & $<0.5$ & 0.6 & 0.6 & 0.6 & $<0.5$ & $<0.5$ & $<0.5$ & 0.5 \\
\hline $\mathrm{Zn}$ & 66 & 63 & 64 & 81 & 66 & 77 & 79 & 83 & 81 & 82 & 74 & 81 & 62 & 61 & 60 \\
\hline $\mathrm{Ni}$ & 136 & 147 & 156 & 125 & 104 & 122 & 115 & 92 & 101 & 101 & 93 & 98 & 116 & 118 & 110 \\
\hline $\mathrm{Cr}$ & 402 & 386 & 441 & 377 & 314 & 466 & 496 & 359 & 335 & 322 & 265 & 282 & 320 & 291 & 293 \\
\hline v & 240 & 233 & 238 & 299 & 286 & 274 & 282 & 285 & 300 & 283 & 271 & 287 & 232 & 234 & 232 \\
\hline $\mathrm{Mg}^{\prime}$ value $^{\mathrm{e}}$ & 0.684 & 0.686 & 0.692 & 0.660 & 0.660 & 0.628 & 0.632 & 0.641 & 0.645 & 0.650 & 0.679 & 0.649 & 0.670 & 0.675 & 0.665 \\
\hline $\mathrm{FeO}$ *d & 8.10 & 8.04 & 8.32 & 8.39 & 8.55 & 8.84 & 8.87 & 9.17 & 9.24 & 10.29 & 7.61 & 8.67 & 8.21 & 7.92 & 8.43 \\
\hline $\mathrm{CaO} / \mathrm{Na}_{2} \mathrm{O}$ & 7.18 & 7.19 & 6.63 & 5.88 & 5.99 & 6.70 & 6.20 & 6.36 & 6.42 & 6.07 & 6.49 & 7.54 & 6.85 & 6.33 & 6.85 \\
\hline
\end{tabular}

ground nonlinearity, interelement interference, and tube contamination by using the methods developed by Norrish and Chappell (1967). Corrections for mass absorption coefficients were based on Reynolds' (1967) Compton scattering method.

The error in the Costa Rica Rift zone basalt analyses (Table 3, Col. 8 ) is expressed as 1 standard deviation of the mean of duplicate (or multiple) determinations. The values were calculated by using the formula $s=\sqrt{(\mathrm{X}-\overline{\mathrm{X}})^{2} /(n-1)}$, where $\mathrm{X}$ and $\overline{\mathrm{X}}$ are observation and mean for replicate analyses of the same element in a single sample and $n$ is the number of samples analyzed. Each element in each sample was measured twice, except for $\mathrm{Nb}$, which was measured 4 to 12 times because of its extremely low concentration. Hence, the error values given refer to the precision of analysis at the concentration levels in the Costa
Rica Rift zone basalts under the given operating conditions. BCR-1 was analyzed as an unknown with each run to provide an estimate of accuracy. Average BCR-1 values are given in Table 3, Column 7, along with 1 standard deviation.

Three samples selected on the basis of XRF data were analyzed for rare earth elements by using isotope dilution techniques on the Stony Brook 12 -in. radius, $90^{\circ}$ sector, NBS design mass spectrometer. The chemical technique used is that of Arth (pers. comm., 1973) and Hanson (1976). The chemical dissolution of samples was performed with graphite crucibles and a lithium metaborate flux. The analytical precision for each REE is better than $2 \%$, except for Gd and Eu, for which it is better than 5\%. Duplicate analyses of Sample 504B-9 were made and are shown in Table 4. An average of these values is used in 
Table 1. (Continued).

\begin{tabular}{|c|c|c|c|c|c|c|c|c|c|c|c|c|c|c|c|c|}
\hline $504 \mathrm{~B}-18$ & $504 \mathrm{~B}-19$ & $504 \mathrm{~B} \cdot 20$ & $504 \mathrm{~B}-21$ & $504 \mathrm{~B}-22$ & $504 \mathrm{~B}-23$ & 504B-24 & $504 \mathrm{~B}-25$ & $504 \mathrm{~B}-26$ & $504 \mathrm{~B}-27$ & $504 \mathrm{~B}-28$ & $504 \mathrm{~B}-29$ & $504 \mathrm{~B}-30$ & 504B-31 & $504 \mathrm{~B}-32$ & $504 \mathrm{~B}-33$ & $504 \mathrm{~B}-34$ \\
\hline $16-3(3)$ & $16-4(130)$ & $17-3$ (15) & $21-3(20)$ & $24-2(78)$ & $24-3(70)$ & $27-1(10)$ & $28-3(3)$ & 29-1 (39) & $32-1$ (29) & $33-1(70)$ & $34-2(50)$ & $35-1(100)$ & $36-1(71)$ & $37-1(104)$ & $38-1(80)$ & $39-1(140)$ \\
\hline 3860.6 & 3863.3 & 3869.7 & 3897.7 & 3923.8 & 3925.2 & 3939.6 & 3951.5 & 3957.9 & 3981.3 & 3990.7 & 4001.0 & 4009.0 & 4017.7 & 4027.0 & 4035.8 & 4045.4 \\
\hline D4 & D3 & D4 & D7 & D5 & DS & D3 & D3 & D3 & Ds & D5 & D7 & D7 & D4 & D4 & D4 & D4 \\
\hline 50.2 & 50.4 & 50.6 & 50.5 & 50.4 & 50.0 & 50.4 & 50,6 & 50.4 & 51.1 & 50.6 & 49.3 & 48.8 & 50.7 & 50.5 & 50.4 & 51.0 \\
\hline 0.91 & 1.00 & 0.94 & 0.76 & 0.87 & 0.85 & 1.00 & 0.96 & 0.98 & 0.87 & 0.91 & 0.81 & 0.77 & 0.88 & 0.92 & 0.89 & 0.93 \\
\hline 15.0 & 16.5 & 16.3 & 18.0 & 16.4 & 16.0 & 15.0 & 16.9 & 14.9 & 16.6 & 16.3 & 16.8 & 17.3 & 15.0 & 15.7 & 15.6 & 16.0 \\
\hline 10.69 & 9.31 & 9.12 & 8.63 & 9.46 & 9.57 & 10.16 & 9.39 & 10.48 & 10.73 & 9.33 & 9.21 & 9.00 & 9.98 & 10.28 & 10.09 & 9.46 \\
\hline 0.19 & 0.17 & 0.17 & 0.13 & 0.17 & 0.17 & 0.18 & 0.19 & 0.23 & 0.13 & 0.14 & 0.15 & 0.12 & 0.14 & 0.14 & 0.14 & 0.15 \\
\hline 8.55 & 8.68 & 8.43 & 8.33 & 8.14 & 8.59 & 8.51 & 7.99 & 8.38 & 8.18 & 8.52 & 8.66 & 9.37 & 8.61 & 8.53 & 8.39 & 7.99 \\
\hline 12.78 & 12.01 & 12.64 & 11.86 & 12.96 & 12.82 & 12.4 & 12.57 & 12.83 & 10.54 & 12.94 & 13.24 & 13.06 & 12.37 & 12.30 & 13.02 & 13.06 \\
\hline 1.9 & 2.1 & 2.1 & 1.9 & 1.9 & 1.8 & 2.0 & 2.2 & 1.9 & 1.9 & 1.9 & 1.9 & 1.9 & 2.1 & 2.1 & 2.1 & 2.1 \\
\hline 0.15 & 0.14 & 0.08 & 0.18 & 0.13 & 0.16 & 0.12 & 0.06 & 0.11 & 0.28 & 0.09 & 0.14 & 0.10 & 0.08 & 0.18 & 0.07 & 0.15 \\
\hline 0.08 & 0.10 & 0.08 & 0.06 & 0.09 & 0.10 & 0.08 & 0.08 & 0.09 & 0.06 & 0.06 & 0.04 & 0.04 & 0.06 & 0.05 & 0.04 & 0.05 \\
\hline 100.45 & 100.41 & 100.46 & 100.35 & 100.52 & 100.06 & 99.85 & 100.94 & 100.30 & 100.39 & 100.79 & 100.25 & 100.46 & 99.92 & 100.70 & 100.74 & 100.89 \\
\hline 3.1 & 1.4 & 0.8 & 1.2 & 0.9 & 1.2 & 1.2 & $<0.5$ & 1.6 & 2.6 & 0.6 & 1.4 & 1.2 & $<0.5$ & 2.1 & 0.7 & 0.8 \\
\hline 57 & 90 & 85 & 59 & 59 & 58 & 68 & 60 & 66 & 59 & 80 & 65 & 76 & 58 & 64 & 58 & 59 \\
\hline 22.9 & 23.3 & 22.7 & 17.2 & 21.2 & 20.1 & 25.6 & 24.3 & 24.8 & 22.1 & 21.7 & 21.1 & 20.0 & 23.4 & 24.8 & 24.0 & 26.5 \\
\hline 16.6 & 16.9 & 14.9 & 14.2 & 15.6 & 18.4 & 15.6 & 16.1 & 16.5 & 14.4 & 14.0 & 14.6 & 14.2 & 15.0 & 13.6 & 15.1 & 15.5 \\
\hline 46 & 56 & 54 & 34 & 43 & 39 & 53 & 46 & 51 & 42 & 54 & 43 & 45 & 44 & 47 & 45 & 47 \\
\hline 0.5 & 0.9 & 0.8 & $<0.5$ & 0.5 & 0.5 & 0.6 & 0.5 & $<0.5$ & 1.0 & 1.0 & $<0.5$ & 0.7 & 1.0 & 0.8 & 1.2 & 0.7 \\
\hline 82 & 77 & 79 & 68 & 74 & 69 & 85 & 83 & 80 & 80 & 75 & 70 & 67 & 66 & 79 & 80 & 83 \\
\hline 102 & 158 & 156 & 142 & 143 & 120 & 110 & 102 & 92 & 106 & 164 & 162 & 191 & 98 & 93 & 108 & 109 \\
\hline 335 & 482 & 492 & 389 & 415 & 412 & 343 & 319 & 314 & 293 & 463 & 436 & 404 & 322 & 417 & 404 & 371 \\
\hline 296 & 252 & 243 & 244 & 244 & 232 & 296 & 281 & 286 & 273 & 241 & 239 & 213 & 270 & 271 & 283 & 288 \\
\hline 0.638 & 0.672 & 0.670 & 0.680 & 0.654 & 0.664 & 0.648 & 0.652 & 0.638 & 0.627 & 0.668 & 0.674 & 0.696 & 0.655 & 0.646 & 0.647 & 0.650 \\
\hline 9.62 & 8.38 & 8.21 & 7.77 & 8.51 & 8.61 & 9.14 & 8.45 & 9.43 & 9.66 & 8.40 & 8.29 & 8.10 & 8.98 & 9.25 & 9.08 & 8.51 \\
\hline 6.73 & 5.72 & 6.02 & 6.24 & 6.82 & 7.12 & 6.20 & 5.71 & 6.75 & 5.55 & 6.81 & 6.97 & 5.68 & 5.89 & 5.86 & 6.20 & 6.22 \\
\hline
\end{tabular}

Table 1. (Continued).

\begin{tabular}{|c|c|c|c|c|c|c|c|c|c|c|c|c|c|c|c|}
\hline $504 \mathrm{~B}-50$ & 504B-51 & $504 \mathrm{~B}-52$ & $504 \mathrm{~B}-53$ & 504B- 54 & $504 \mathrm{~B}-55$ & 504B- 56 & 504B- 57 & 504B-58 & 504B-59 & $504 \mathrm{~B}-60$ & $504 \mathrm{~B}-61$ & $504 \mathrm{~B}-62$. & $504 \mathrm{~B}-63$ & $504 \mathrm{~B}-64$ & $504 \mathrm{~B}-65$ \\
\hline $54-1(90)$ & $55-2(34)$ & $56-2(62)$ & $57-1(36)$ & $58-1(92)$ & $59-1(27)$ & $60-2$ (1) & $61-1(88)$ & $62-2(21)$ & $63-1(122)$ & 64-2 (1) & $65-1(56)$ & $66-2(99)$ & $67-1(61)$ & 68-1 (1) & $70-1(95)$ \\
\hline${ }_{\mathrm{M}}^{4165.9}$ & $\underset{\mathrm{M}}{4171.8}$ & $\frac{4181.1}{\mathrm{M}}$ & $\begin{array}{c}4188.0 \\
M\end{array}$ & $\begin{array}{l}4197.9 \\
\text { D3 }\end{array}$ & $\begin{array}{c}4206.3 \\
\mathrm{D} 3\end{array}$ & $\begin{array}{c}4216.5 \\
D_{3} 3\end{array}$ & $\begin{array}{c}4224.9 \\
\text { D3 }\end{array}$ & $\begin{array}{c}4230.2 \\
D 3\end{array}$ & $\begin{array}{c}4238.7 \\
\mathrm{D} 3\end{array}$ & $\begin{array}{c}42480^{\prime} \\
\mathrm{D} 1\end{array}$ & $\begin{array}{c}4256.1 \\
D 4\end{array}$ & $\begin{array}{c}4267.0 \\
\mathrm{D} 2\end{array}$ & $\begin{array}{l}4274.1 \\
\mathrm{D} 2\end{array}$ & $\begin{array}{c}4282.5 \\
D 2\end{array}$ & $\begin{array}{l}4301.5 \\
\mathrm{D} 2\end{array}$ \\
\hline & & & & & & & & & & & & & & & \\
\hline $\begin{array}{l}30.4 \\
1.39\end{array}$ & $\begin{array}{l}30.3 \\
1.35\end{array}$ & 1.36 & $\begin{array}{l}30.3 \\
1.37\end{array}$ & $\begin{array}{r}30.96 \\
0.96\end{array}$ & $\begin{array}{l}50.3 \\
0.99\end{array}$ & $\begin{array}{l}50.3 \\
0.97\end{array}$ & $\begin{array}{r}49.6 \\
1.03\end{array}$ & $\begin{array}{r}50.7 \\
1.02\end{array}$ & $\begin{array}{l}30.5 \\
1.04\end{array}$ & $\begin{array}{r}1.1 \\
1.19\end{array}$ & $\begin{array}{c}50.6 \\
0.94\end{array}$ & $\begin{array}{c}1.3 \\
1.08\end{array}$ & $\begin{array}{c}50.3 \\
1.03\end{array}$ & $\begin{array}{c}50.3 \\
1.03\end{array}$ & $\begin{array}{l}50.9 \\
1.07\end{array}$ \\
\hline 15.4 & 15.1 & 15.3 & 15.2 & 15.8 & 15.6 & 15.4 & 15.4 & 15.3 & $\begin{array}{l}15.64 \\
15\end{array}$ & 14.4 & 15.5 & $\begin{array}{l}14.7 \\
140\end{array}$ & 15.1 & 15.0 & 14.6 \\
\hline 9.54 & $\begin{array}{l}10.12 \\
10.12\end{array}$ & 10.09 & 10.05 & $\begin{array}{l}13.8 \\
10.22\end{array}$ & $\begin{array}{l}9.8 \\
9.84\end{array}$ & 9.59 & $\begin{array}{l}13.4 \\
10.64\end{array}$ & $\begin{array}{l}\text { 13.10 } \\
11.30\end{array}$ & $\begin{array}{l}15.6 \\
10.32\end{array}$ & $\begin{array}{l}14.4 \\
11.62\end{array}$ & 9.75 & $\begin{array}{l}14.09 \\
11.09\end{array}$ & $\begin{array}{l}10.30 \\
10.30\end{array}$ & $\begin{array}{l}10.35 \\
10.35\end{array}$ & $\begin{array}{l}14.6 \\
10.88\end{array}$ \\
\hline 0.17 & 0.17 & 0.19 & 0.17 & 0.17 & 0.14 & 0.15 & 0.16 & 0.13 & 0.15 & 0.19 & 0.16 & 0.15 & 0.18 & 0.18 & 0.22 \\
\hline 7.92 & 8.09 & 8.53 & 8.15 & 8.21 & 10.23 & 8.85 & 8.11 & 10.94 & 8.46 & 8.04 & 8.60 & 8.22 & 8.74 & 8.91 & 8.28 \\
\hline 12.80 & 12.71 & 12.11 & 12.53 & 12.73 & 11.28 & 12.49 & 12.89 & 9.49 & 12.69 & 11.83 & 12.75 & 12.18 & 12.38 & 11.93 & $\begin{array}{l}0.260 \\
12.18\end{array}$ \\
\hline 2.4 & 2.4 & & 2.5 & 2.0 & 2.1 & 2.2 & 1.8 & 1.7 & 1.8 & 2.1 & 2.0 & 2.2 & 2.0 & 2.0 & 2.0 \\
\hline 0.06 & & & & & 0.07 & 0.07 & 0.04 & 0.12 & 0.05 & 0.06 & 0.04 & 0.04 & 0.03 & 0.04 & 0.05 \\
\hline 0.12 & 0.14 & 0.12 & 0.12 & 0.05 & 0.05 & 0.07 & 0.07 & 0.06 & 0.06 & 0.07 & 0.05 & 0.06 & 0.05 & 0.07 & 0.07 \\
\hline 100.20 & 100.44 & 100.96 & 100.46 & 100.89 & 100.80 & 100.09 & 99.74 & 100.56 & 100.58 & 100.60 & 100.39 & 101.02 & 100.31 & 99.81 & 100.05 \\
\hline 0.6 & $<0.5$ & $<0.5$ & $<0.5$ & $<0.5$ & $<0.5$ & $<0.5$ & 0.8 & 2.0 & 0.6 & $<0.5$ & 0.7 & $<0.5$ & $<0.5$ & $<0.5$ & $<0.5$ \\
\hline 106 & 107 & 104 & 104 & 56 & 70 & 75 & so & 58 & 51.0 & 55 & 64 & 55 & 57 & 56 & 66 \\
\hline 32.0 & 30.6 & 31.2 & 30.8 & 24.5 & 22.9 & 24.0 & 27.2 & 25.0 & 27.4 & 29.0 & 24.1 & 27.4 & 26.5 & 26.1 & 22.8 \\
\hline 15.7 & 16.6 & 16.7 & 15.6 & 15.9 & 15.5 & 15.4 & 15.7 & 13.7 & 15.3 & 16.9 & 15.0 & 15.6 & 15.7 & 15.2 & 14.4 \\
\hline 104 & 103 & 103 & 104 & 48 & 54 & 55 & 54 & 51 & 54 & 60 & 51 & 52 & 50 & 50 & 47 \\
\hline 2.2 & 1.9 & 2.6 & 2.9 & 0.8 & 0.7 & 0.7 & 1.1 & 0.6 & 0.9 & 0.8 & 1.2 & 1.0 & 0.6 & $<0.5$ & $<0.5$ \\
\hline 82 & 74 & 82 & 78 & 83 & 71 & 69 & 88 & 84 & 85 & 89 & 82 & 83 & 93 & 87 & 77 \\
\hline 97 & 99 & 94 & 96 & 97 & 138 & 132 & 129 & 119 & 12 & 75 & 105 & 79 & 101 & 96 & 132 \\
\hline 275 & 216 & 282 & 253 & 248 & 299 & 321 & 332 & 313 & & 156 & & & & 245 & 424 \\
\hline 322 & 291 & 316 & 312 & 298 & 262 & 251 & 328 & 317 & 311 & 336 & 299 & 319 & 325 & 324 & 257 \\
\hline 0.646 & 0.638 & 0.650 & 0.641 & 0.639 & 0.696 & 0.670 & 0.627 & 0.684 & 0.643 & 0.604 & 0.660 & 0.620 & 0.647 & 0.655 & 0.626 \\
\hline 8.58 & 9.11 & 9.08 & 9.04 & 9.20 & 8.85 & 8.63 & 9.57 & 9.99 & 9.29 & 10.46 & 8.77 & 9.98 & 9.45 & 9.31 & 9.79 \\
\hline 5.33 & 5.29 & 4.84 & 5.01 & 6.37 & 5.37 & 5.68 & 7.16 & 5.58 & 7.05 & 5.63 & 6.38 & 5.54 & 6.19 & 5.97 & 6.41 \\
\hline
\end{tabular}

the figures and discussion. Leedey chondrite values (Masuda et al., 1973) divided by 1.2 are used for normalizing purposes in this study (Langmuir et al., 1977).

Seven samples were melted in a 1-atm. Deltech furnace with $\mathrm{fO}_{2}$ held approximately equal to $\mathrm{QFM}\left(\mathrm{CO}=4.75 \%, \mathrm{CO}_{2}=95.25 \%\right)$ at various temperatures (Table 6). Thirty- to $50-\mathrm{mg}$ aliquots of pressed powder bound with polyvinyl alcohol were sintered onto presaturated $\mathrm{PtFe}(\mathrm{Fe} \cong 10 \%)$ 0.004-in.-diameter wire loops and held in the furnace for 20 to $239 \mathrm{hr}$. Temperature was measured with a $\mathrm{Pt}_{90} \mathrm{Rh}_{10}$ thermocouple calibrated against the melting point of gold. Samples were submersed rapidly (1 s) in water introduced at the base of the furnace while still in the gas stream (Walker et al., 1979). Run products were broken, mounted in epoxy, and examined petrographically in reflected light.

\section{BASALT CHEMISTRY}

Two types of magma are evident among the Costa Rica Rift zone basalts: a rare basalt (Group M) that is similar to the basalt called Type I by Bryan et al. (1976) and a basalt that is highly depleted relative to most Type I and II basalts (Group D). Most of the samples studied here belong to Group D (Tables 1-3). The Group M basalts, which occur in a few thin layers that are repeated in the Hole 504B stratigraphic column (Fig. 3), are petrographically and chemically very close to or at multiple saturation. They exhibit moderate $\mathrm{Mg}^{\prime}$ values $(0.64)$ 
Table 2. Chemical composition of basalts from Hole 501 .

\begin{tabular}{|c|c|c|c|c|c|c|c|c|c|c|c|c|c|c|c|c|c|c|}
\hline Sample & $501-1$ & $501-2$ & $501-3$ & $501-4$ & $501-5$ & $501-6$ & $501-7$ & $501-8$ & $501-9$ & $501-10$ & $501-11$ & $501-12$ & $501-13$ & $501-14$ & $501-15$ & $501-16$ & $501-17$ & $501-18$ \\
\hline Core-Section ${ }^{\mathrm{a}}$ & $10-1(52)$ & $11-1(87)$ & $12-1(112)$ & $12-1(143)$ & $14-2(136)$ & $14-3(61)$ & $14-3(146)$ & $15-1(3)$ & $15-2(66)$ & $16-1(72)$ & $17-3(132)$ & $18-1(3)$ & $18-1(90)$ & $18-2(102)$ & 19-1 (83) & $20-1(47)$ & $20-3(120)$ & $20-4$ (2) \\
\hline Depth $(m)^{b}$ & 3721.5 & 3721.7 & 3731.1 & 3731.4 & 3742.4 & 3743.6 & 3744.5 & 3749.0 & 3751.2 & 3758.7 & 3766.3 & 3767.0 & 3767.9 & 3769.5 & 3776.8 & 3785.5 & 3789.2 & 3789.5 \\
\hline Basalt Type ${ }^{\mathrm{C}}$ & D1 & D1 & D1 & D1 & D1 & D1 & D4 & D4 & D4 & D4 & D4 & $\mathrm{D} 2$ & D4 & $\mathrm{D} 2$ & D3 & D3 & D3 & D3 \\
\hline \multicolumn{19}{|c|}{ Major elements (wt.\%) } \\
\hline $\mathrm{SiO}_{2}$ & 50.5 & 51.1 & 50.5 & 50.1 & 49.7 & 51.2 & 50.5 & 49.7 & 51.6 & 49.9 & 50.4 & 51.2 & 49.8 & 50.7 & 49.8 & 50.2 & 50.3 & 51.0 \\
\hline $\mathrm{TiO}_{2}$ & 1.10 & 1.13 & 1.12 & 1.11 & 1.10 & 1.08 & 0.92 & 0.92 & 0.96 & 0.92 & 0.95 & 1.04 & 0.92 & 1.08 & 0.99 & 0.97 & 0.96 & 0.92 \\
\hline $\mathrm{Al}_{2} \mathrm{O}_{3}$ & 15.1 & 15.4 & 15.5 & 15.1 & 14.2 & 15.2 & 16.7 & 16.5 & 16.1 & 16.5 & 16.3 & 17.8 & 15.9 & 14.9 & 16.8 & 15.3 & 15.7 & 15.6 \\
\hline $\mathrm{Fe}_{2} \mathrm{O}_{3} * \mathrm{~d}$ & 11.10 & 11.3 & 10.64 & 10.75 & 12.33 & 10.09 & 9.44 & 9.66 & 10.16 & 9.39 & 9.89 & 9.05 & 10.34 & 10.63 & 9.34 & 10.82 & 10.23 & 9.61 \\
\hline Mno & 0.22 & 0.17 & 0.19 & 0.18 & 0.19 & 0.19 & 0.19 & 0.20 & 0.11 & 0.20 & 0.18 & 0.14 & 0.20 & 0.20 & 0.14 & 0.18 & 0.18 & 0.18 \\
\hline $\mathrm{MgO}$ & 7.96 & 8.86 & 7.94 & 8.09 & 7.71 & 8.43 & 8.12 & 7.71 & 15.25 & 7.68 & 7.86 & 8.03 & 8.02 & 7.85 & 8.52 & 8.95 & 8.25 & 8.05 \\
\hline $\mathrm{CaO}$ & 12.29 & 10.20 & 12.00 & 12.27 & 11.78 & 12.00 & 12.67 & 13.24 & 2.57 & 13.34 & 12.43 & 11.29 & 12.67 & 12.19 & 11.52 & 11.86 & 12.27 & 12.72 \\
\hline $\mathrm{Na}_{2} \mathrm{O}$ & 1.8 & 2.1 & 2.2 & 2.2 & 2.0 & 2.2 & 1.8 & 1.9 & 1.1 & 1.8 & 1.9 & 2.2 & 1.9 & 1.8 & 2.1 & 1.8 & 1.7 & 2.0 \\
\hline $\mathrm{K}_{2} \mathrm{O}$ & 0.18 & 0.49 & 0.24 & 0.15 & 0.41 & 0.06 & 0.13 & 0.08 & 0.70 & 0.10 & 0.15 & 0.23 & 0.09 & 0.30 & 0.19 & 0.19 & 0.13 & 0.14 \\
\hline $\mathrm{P}_{2} \mathrm{O}_{5}$ & 0.08 & 0.07 & 0.09 & 0.10 & 0.09 & 0.11 & 0.08 & 0.09 & 0.03 & 0.08 & 0.08 & 0.08 & 0.08 & 0.10 & 0.10 & 0.08 & 0.09 & 0.07 \\
\hline Total & 100.33 & 100.82 & 100.42 & 100.05 & 99.51 & 100.56 & 100.55 & 100.00 & 98.58 & 99.91 & 100.14 & 101.06 & 99.92 & 99.75 & 99.50 & 100.35 & 99.81 & 100.29 \\
\hline \multicolumn{19}{|c|}{ Trace elements (ppm) } \\
\hline $\mathrm{Rb}$ & 2.1 & 4.5 & 4.3 & 2.8 & 9.1 & 0.6 & 1.2 & 1.0 & 4.3 & 1.6 & 1.0 & 1.6 & 0.9 & 5.7 & 2.4 & 3.4 & 1.2 & 1.8 \\
\hline $\mathrm{Sr}$ & 69 & 75 & 73 & 71 & 71 & 69 & 71 & 70 & 70 & 67 & 58 & 71 & 55 & 57 & 67 & 57 & 59 & 54 \\
\hline $\mathrm{Y}$ & 27.2 & 25.9 & 28.5 & 28.1 & 27.4 & 27.4 & 22.9 & 23.0 & 6.9 & 22.9 & 23.3 & 24.9 & 24.6 & 29.1 & 25.9 & 25.9 & 27.3 & 25.1 \\
\hline $\mathrm{Ga}$ & 17.4 & 14.2 & 17.1 & 17.2 & 17.0 & 16.7 & 15.3 & 15.6 & 6.8 & 15.8 & 15.6 & 16.0 & 16.0 & 17.4 & 17.3 & 15.8 & 16.2 & 16.6 \\
\hline $\mathrm{Zr}$ & 55 & 58 & 57 & 56 & 56 & 55 & 48 & 47 & 46 & 48 & 44 & 45 & 43 & 51 & 45 & 43 & 44 & 43 \\
\hline $\mathrm{Nb}$ & $<0.5$ & $<0.5$ & 0.8 & 0.8 & 0.7 & 1.1 & $<0.5$ & $<0.5$ & 0.6 & $<0.5$ & 0.6 & $<0.5$ & 0.9 & 0.6 & 1.0 & $<0.5$ & $<0.5$ & 0.7 \\
\hline $\mathrm{Zn}$ & 90 & 77 & 94 & 89 & 87 & 93 & 77 & 75 & 53 & 74 & 83 & 84 & 85 & 104 & 88 & 94 & 87 & 83 \\
\hline & 88 & 80 & 94 & 94 & 79 & 127 & 133 & 133 & 101 & 145 & 115 & 115 & 108 & 99 & 102 & 106 & 108 & 116 \\
\hline $\mathrm{Cr}$ & 236 & 218 & 215 & 223 & 183 & 183 & 451 & 439 & 420 & 426 & 309 & 295 & 275 & 199 & 273 & 261 & 279 & 259 \\
\hline v & 329 & 328 & 348 & 327 & 342 & 339 & 263 & 254 & 253 & 251 & 305 & 318 & 301 & 326 & 316 & 300 & 310 & 310 \\
\hline $\mathrm{Mg}^{\prime}$ value ${ }^{\mathrm{e}}$ & 0.612 & 0.633 & 0.622 & 0.624 & 0.579 & 0.648 & 0.654 & 0.637 & 0.768 & 0.642 & 0.636 & 0.661 & 0.631 & 0.619 & 0.668 & 0.645 & 0.640 & 0.648 \\
\hline $\mathrm{FeO} * \mathrm{~d}$ & 9.99 & 10.17 & 9.57 & 9.67 & 11.10 & 9.08 & 8.49 & 8.69 & 9.14 & 8.45 & 8.90 & 8.14 & 9.30 & 9.57 & 8.40 & 9.74 & 9.21 & 8.65 \\
\hline $\mathrm{CaO} / \mathrm{Na}_{2} \mathrm{O}$ & 6.83 & 4.86 & 5.45 & 5.58 & 5.89 & 5.45 & 7.04 & 6.97 & 2.34 & 7.41 & 6.54 & 5.13 & 6.67 & 6.77 & 5.49 & 6.59 & 7.22 & 6.36 \\
\hline
\end{tabular}

${ }^{a}$ Level (in parentheses) in $\mathrm{cm}$.

c Basalt type refers to chemical units discussed in the text.

$\mathrm{d}^{\mathrm{Fe}} \mathrm{O}_{3}{ }^{*}$ and $\mathrm{FeO} *$ refer to total $\mathrm{Fe}$ expressed as $\mathrm{Fe}_{2} \mathrm{O}_{3}$ or $\mathrm{FeO}$ 
Table 3. Chemical composition of basalt samples from Holes 504A, 505A, and 505B.

\begin{tabular}{|c|c|c|c|c|c|c|c|c|}
\hline Sample & $504 \mathrm{~A}-1$ & $505 \mathrm{~A}-1$ & 505B-1 & $505 \mathrm{~B}-2$ & $505 \mathrm{~B}-3$ & $505 \mathrm{~B}-4$ & $\mathrm{BCR}-1^{\mathrm{a}}$ & \\
\hline $\begin{array}{l}\text { Hole-Core-Section } \\
\text { (level in } \mathrm{cm} \text { ) }\end{array}$ & $\begin{array}{c}504 \mathrm{~A}-6-1 \\
(47)\end{array}$ & $\begin{array}{l}505 A-25-1 \\
\text { (2) }\end{array}$ & $\begin{array}{l}\text { 505B-2-1 } \\
(109)\end{array}$ & $\begin{array}{c}\text { S0sB-2-2 } \\
\text { (9) }\end{array}$ & $\begin{array}{l}\text { 505B-3-1 } \\
(27)\end{array}$ & $\begin{array}{c}505 \mathrm{~B}-6-2 \\
(44)\end{array}$ & $\bar{z}$ & \\
\hline Basalt Typec & & & D4 & D4 & D4 & D4 & - & $\begin{array}{l}\text { CRRZ } \\
\text { Error }\end{array}$ \\
\hline \multicolumn{9}{|c|}{ Major elements (wt.\%) } \\
\hline $\mathrm{SiO}_{2}$ & 51.3 & 49.4 & 50.1 & 49.1 & 49.8 & 49.8 & $54.6(0.4)$ & 0.1 \\
\hline $\mathrm{TiO}_{2}$ & 1.08 & 0.77 & 0.95 & 0.95 & 0.98 & 0.95 & $2.24(0.01)$ & 0.02 \\
\hline $\mathrm{Al}_{2} \mathrm{O}_{3}$ & 15.0 & 17.4 & 16.3 & 16.0 & 16.2 & 16.1 & $13.5(0.1)$ & 0.1 \\
\hline $\mathrm{Fe}_{2} \mathrm{O}_{3}$, & 10.48 & 8.22 & 9.23 & 9.70 & 9.57 & 9.26 & $13.2(0.1)$ & 0.02 \\
\hline $\mathrm{MnO}$ & 0.18 & 0.13 & 0.14 & 0.16 & 0.16 & 0.16 & $0.18(0.01)$ & 0.01 \\
\hline $\mathrm{MgO}$ & 7.70 & 8.85 & 8.86 & 8.69 & 8.58 & 8.61 & $3.36(0.03)$ & 0.04 \\
\hline $\mathrm{CaO}$ & 12.22 & 13.28 & 12.6 & 12.7 & 12.6 & 12.6 & $6.90(0.04)$ & 0.02 \\
\hline $\mathrm{Na}_{2} \mathrm{O}$ & 2.1 & 2.0 & 2.2 & 2.1 & 2.2 & 2.2 & - & - \\
\hline $\mathrm{K}_{2} \mathrm{O}$ & 0.06 & 0.15 & 0.09 & 0.11 & 0.11 & 0.15 & $1.72(0.01)$ & 0.01 \\
\hline $\mathrm{P}_{2} \mathrm{O}_{5}$ & 0.10 & 0.09 & 0.09 & 0.08 & 0.07 & 0.08 & $0.35(0.02)$ & 0.01 \\
\hline Total & 100.20 & 100.29 & 100.56 & 99.59 & 100.27 & 99.91 & - & - \\
\hline
\end{tabular}

Trace elements (ppm)

\begin{tabular}{|c|c|c|c|c|c|c|c|c|}
\hline $\mathbf{R b}$ & 0.6 & 1.5 & 0.6 & 1.8 & 1.6 & 2.4 & $47.1(0.1)$ & 0.2 \\
\hline Sr & 67 & 78 & 73 & 76 & 75 & 76 & 331 (1) & 1 \\
\hline $\mathrm{Y}$ & 25.8 & 18.3 & 23.5 & 23.6 & 24.4 & 23.3 & $33.7(0.1)$ & 0.3 \\
\hline Ga & 18.6 & 14.8 & 13.8 & 15.3 & 15.3 & 15.1 & $22.5(0.2)$ & 0.8 \\
\hline $\mathrm{Zr}$ & 54 & 43 & 54 & 56 & 56 & 54 & 192 (1) & 1 \\
\hline $\mathrm{Nb}$ & $<0.5$ & 1.0 & 1.1 & 0.8 & 1.9 & 0.8 & $12.2(0.1)$ & 0.5 \\
\hline $\mathrm{Zn}$ & 84 & 63 & 72 & 76 & 76 & 73 & 150 (1) & 1 \\
\hline $\mathrm{Ni}$ & 84 & 176 & 152 & 145 & 153 & 174 & $-\quad-$ & 1 \\
\hline $\mathrm{Cr}$ & 196 & 497 & 397 & 428 & 434 & 413 & $-\quad-$ & 2 \\
\hline v & 321 & 205 & 235 & 244 & 252 & 233 & 370 (1) & 3 \\
\hline $\mathrm{Mg}^{\prime}$ value $^{\mathrm{e}}$ & 0.618 & 0.703 & 0.679 & 0.663 & 0.664 & 0.672 & - & - \\
\hline $\mathrm{FeO} \cdot \mathrm{d}$ & 9.43 & 7.40 & 8.31 & 8.73 & 8.61 & 8.33 & - & - \\
\hline $\mathrm{CaO} / \mathrm{Na}_{2} \mathrm{O}$ & 5.82 & 6.64 & 5.73 & 6.05 & 5.73 & 5.73 & - & - \\
\hline
\end{tabular}

a BCR-1 was run as an internal standard for both major and trace element XRF analyses. Values represent mean and 1 standard deviation (in parentheses).

CRRZ error refers to the analytical error for the Costa Rica Rift zone basalts which was calculated by using the formula $s=\sqrt{(\mathrm{X}-\overline{\mathrm{X}})^{2} /(n-1)}($ see text).

Basalt type refers to chemical units discussed in the text.

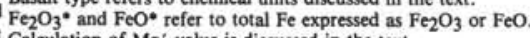

Table 4. REE abundances.

\begin{tabular}{|c|c|c|c|c|c|}
\hline & \multicolumn{4}{|c|}{ Sample } & \multirow[b]{2}{*}{$\mathrm{N}^{\mathrm{a}}$} \\
\hline & 504B-21 & 504B-9A & 504B-9B & 504B-53 & \\
\hline \multicolumn{6}{|c|}{ Abundances $(\mathrm{ppm})$ in samples of - } \\
\hline $\mathrm{Ce}$ & 2.91 & 3.92 & 4.05 & 10.6 & - \\
\hline $\mathrm{Nd}$ & 3.49 & 4.57 & 4.71 & 9.48 & - \\
\hline Sm & 1.49 & 1.83 & 1.88 & 3.16 & - \\
\hline Eu & 0.615 & 0.714 & 0.717 & 1.16 & - \\
\hline $\mathrm{Gd}$ & 2.26 & 2.72 & 2.67 & 4.14 & - \\
\hline Dy & 3.17 & 3.84 & 3.80 & 5.39 & - \\
\hline $\mathrm{Er}$ & 2.14 & 2.55 & 2.56 & 3.52 & - \\
\hline $\mathrm{Yb}$ & 2.15 & 2.50 & 2.49 & 3.37 & - \\
\hline \multicolumn{6}{|c|}{$($ ppm sample $\times 1.2)$} \\
\hline \multicolumn{6}{|c|}{ Normalized abundances $\left(\frac{\mathrm{ppm}}{\mathrm{ppm} \text { Leedey }}\right)$} \\
\hline $\mathrm{Ce}$ & 3.58 & 4.82 & 4.98 & 12.98 & 0.813 \\
\hline Nd & 5.85 & 7.66 & 7.89 & 15.87 & 0.597 \\
\hline Sm & 7.74 & 9.54 & 9.79 & 16.47 & 0.192 \\
\hline $\mathrm{Eu}$ & 8.51 & 9.89 & 9.93 & 16.06 & 0.0722 \\
\hline Gd & 8.70 & 10.49 & 10.30 & 15.99 & 0.259 \\
\hline Dy & 9.76 & 11.80 & 11.69 & 16.59 & 0.325 \\
\hline $\mathrm{Er}$ & 10.03 & 11.96 & 12.02 & 16.51 & 0.213 \\
\hline $\mathrm{Yb}$ & 10.34 & 12.00 & 11.97 & 16.20 & 0.208 \\
\hline Basalt type ${ }^{b}$ & D7 & D4 & D4 & M & \\
\hline $\mathrm{Ce} / \mathrm{Sm}$ & 0.462 & 0.505 & 0.509 & 0.788 & - \\
\hline $\mathrm{Ce} / \mathrm{Yb}$ & 0.346 & 0.402 & 0.416 & 0.801 & - \\
\hline
\end{tabular}

${ }^{a} \mathrm{~N}$ refers to chondrite normalizing value.

$\mathrm{b}$ Basalt type refers to chemical units discussed in the text.

and moderate abundances of $\mathrm{TiO}_{2}(1.3-1.4 \%), \mathrm{Na}_{2} \mathrm{O}$ (2.4-2.5\%), Y (30-31 ppm), Zr (103-104 ppm), Nb (2-3 $\mathrm{ppm})$, and $\mathrm{Sr}$ (104-107 ppm). All of these values are well within the range exhibited by Type I basalts. Low abundances of $\mathrm{Cr}$ (216-282 ppm) and $\mathrm{Ni}$ (94-99 ppm) and mod-

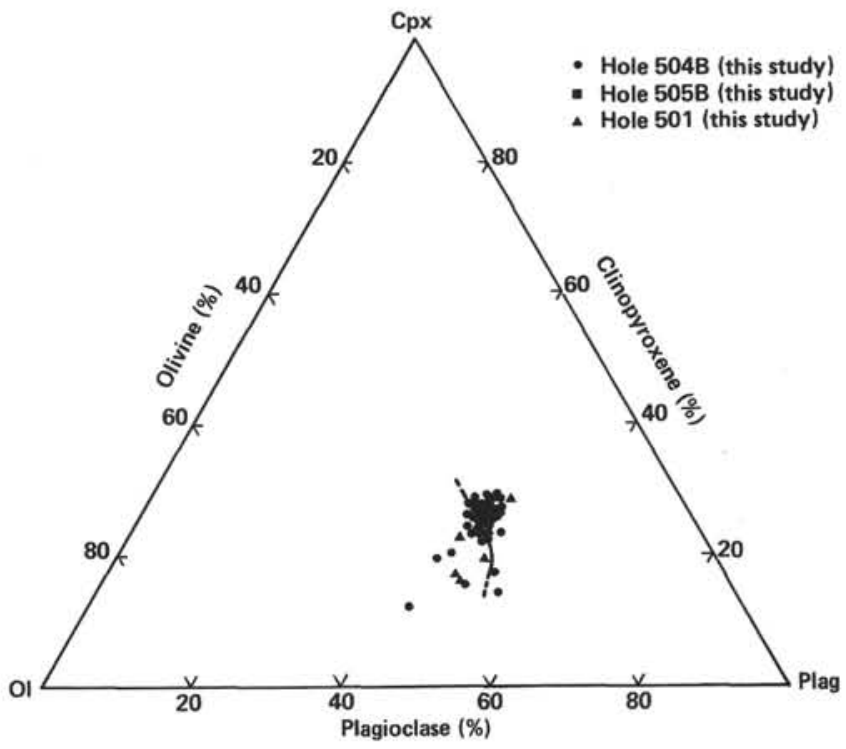

Figure 1. Projection from quartz onto normalized olivine-clinopyroxene-plagioclase surface (Walker et al., 1979). The olivine-plagioclase cotectic is based on the data presented in Shido et al. (1971) and is only a reference line. The Costa Rica Rift zone basalts are moderately evolved, with olivine, plagioclase, and often clinopyroxene on the liquidus.

erate abundances of $\mathrm{MgO}(7.9-8.5 \%)$ and $\mathrm{FeO}^{*}$ (8.6$9.1 \%$ ) indicate a substantial history of fractional crystallization (O'Hara, 1968), particularly of olivine and spinel. The Group $\mathrm{M}$ samples have low melting points $\left(\sim 1210^{\circ} \mathrm{C}\right.$, Fig. 4$)$ and are calculated to be in equilib- 
Table 5. Basalt group averages.

\begin{tabular}{|c|c|c|c|c|c|c|c|c|}
\hline Sample Designation & M & D1 & D2 & D3 & D4 & DS & D6 & D7 \\
\hline Number of Samples & 4 & 3 & 4 & 11 & 28 & 4 & 5 & 6 \\
\hline \multicolumn{9}{|c|}{ Major elements (wt.\%) } \\
\hline $\mathrm{SiO}_{2}$ & $50.4(1)$ & $50.8(2)$ & 50.7 (2) & 50.4 (1) & 50.4 (1) & 50.5 (2) & $50.7(3)$ & $49.8(3)$ \\
\hline $\mathrm{TiO}_{2}$ & 1.37 (1) & $1.13(3)$ & 1.05 (1) & 1.00 (1) & 0.92 (1) & 0.88 (1) & 0.82 (1) & 0.78 (1) \\
\hline $\mathrm{Al}_{2} \mathrm{O}_{3}$ & 15.3 (1) & $15.8(10)$ & 14.9 (1) & 15.7 (2) & 15.8 (1) & 16.3 (1) & 16.3 (2) & 16.9 (3) \\
\hline $\mathrm{Fe}_{2} \mathrm{O}_{3}{ }^{\circ}$ & 9.95 (14) & $10.9(5)$ & 10.7 (2) & 10.1 (2) & $9.86(10)$ & $9.77(32)$ & 9.08 (21) & $9.01(9)$ \\
\hline $\mathrm{MnO}^{\circ}$ & 0.18 (1) & 0.17 (2) & 0.18 (1) & 0.17 (1) & 0.16 (1) & 0.15 (1) & 0.14 (1) & 0.14 (1) \\
\hline $\mathrm{MgO}$ & 8.17 (13) & 7.79 (17) & $8.54(17)$ & $8.77(27)$ & $8.26(8)$ & $8.36(12)$ & $8.28(7)$ & 8.92 (17) \\
\hline $\mathrm{CaO}$ & $12.5(2)$ & $11.5(5)$ & 12.2 (1) & 12.1 (3) & $12.8(1)$ & $12.3(6)$ & 13.0 (1) & $12.8(2)$ \\
\hline $\mathrm{Na}_{2} \mathrm{O}$ & $2.5(1)$ & $2.2(1)$ & 2.1 (1) & 2.0 (1) & $2.0(1)$ & 1.9 (1) & 1.9 (1) & 1.9 (1) \\
\hline $\mathrm{K}_{2} \mathrm{O}$ & 0.06 (1) & $0.26(12)$ & 0.04 (1) & 0.10 (2) & 0.12 (1) & 0.17 (4) & 0.06 (1) & 0.10 (2) \\
\hline $\mathrm{P}_{2} \mathrm{O}_{5}$ & 0.13 (1) & $0.10(1)$ & $0.06(1)$ & 0.07 (1) & 0.07 (1) & 0.08 (1) & 0.05 (1) & 0.04 (1) \\
\hline Total & 100.56 & 100.65 & 100.47 & 100.41 & 100.39 & 100.41 & 100.33 & 100.39 \\
\hline \multicolumn{9}{|l|}{ Trace elements (ppm) } \\
\hline $\mathrm{Rb}$ & $<0.5$ & $4.2(16)$ & $<0.5$ & $1.2(4)$ & $1.5(3)$ & $1.3(4)$ & $<0.5$ & $1.0(1)$ \\
\hline Sr & 105 (1) & $73(8)$ & 59 (2) & $66(4)$ & 65 (1) & $64(5)$ & $57(3)$ & 64 (2) \\
\hline $\mathbf{Y}$ & $31.2(3)$ & $24.2(28)$ & 25.7 (9) & $24.8(4)$ & 23.5 (2) & $21.2(4)$ & 21.7 (4) & $19.8(5)$ \\
\hline $\mathrm{Ga}$ & $16.2(3)$ & 16.7 (1) & 15.2 (3) & 15.8 (2) & 15.4 (2) & 15.7 (9) & 15.5 (3) & 14.5 (1) \\
\hline $\mathrm{Zr}$ & 103.7 (4) & 55.8 (14) & 49.7 (8) & $52.1(8)$ & $46.9(6)$ & $44.4(28)$ & $40.1(5)$ & 40.2 (15) \\
\hline $\mathrm{Nb}$ & $2.4(2)$ & $0.6(1)$ & 0.5 (2) & 0.7 (1) & $0.6(1)$ & 0.8 (2) & $<0.5$ & 0.6 (1) \\
\hline $\mathrm{Zn}$ & 79 (2) & 87 (2) & 85 (3) & $80(2)$ & 78 (1) & $.75(2)$ & $68(4)$ & 66 (1) \\
\hline $\mathrm{Ni}$ & $97(2)$ & 77 (1) & $102(10)$ & $118(6)$ & $115(4)$ & 133 (11) & 107 (4) & $156(7)$ \\
\hline $\mathrm{Cr}$ & 257 (13) & 263 (58) & 292 (39) & 333 (17) & 386 (ii) & $396(32)$ & $290(8)$ & 410 (9) \\
\hline $\mathrm{v}$ & 310 (12) & $320(8)$ & 306 (15) & $286(8)$ & $276(5)$ & $248(8)$ & $251(10)$ & $235(4)$ \\
\hline $\mathrm{Mg}^{\prime}$ & $0.644(3)$ & $0.612(7)$ & $0.637(8)$ & $0.654(7)$ & 0.648 (3) & $0.653(9)$ & $0.668(5)$ & $0.685(3)$ \\
\hline $\mathrm{CaO} / \mathrm{Na}_{2} \mathrm{O}$ & 5.00 & 5.23 & 5.81 & 6.05 & 6.40 & 6.47 & 6.84 & 6.74 \\
\hline
\end{tabular}

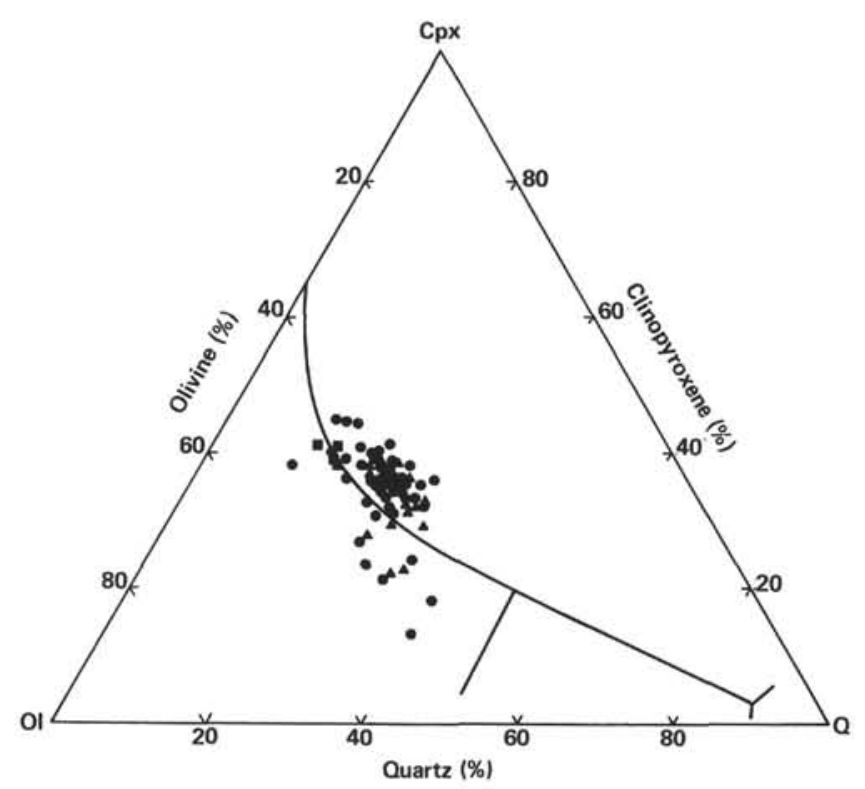

Figure 2. Projection from plagioclase onto normalized olivine-clinopyroxene-silica diagram. A reference olivine-plagioclase-clinopyroxene multiple saturation curve (Walker et al., 1979) is shown. This diagram supports the moderate evolution of the Costa Rica Rift zone basalts (most are close to or at multiple saturation). Symbols as in Figure 1.

rium with plagioclase compositions that are typical of mid-ocean-ridge basalts (MORB) $\left(\mathrm{An}_{70-75}\right)$ (Drake, 1976).

Almost all of the Costa Rica Rift zone samples belong to the depleted Group D. They exhibit low abundances of $\mathrm{TiO}_{2}(0.75-1.2 \%), \mathrm{Na}_{2} \mathrm{O}(1.7-2.3 \%)$, and $\mathrm{K}_{2} \mathrm{O}$ $(0.03-0.5 \%)$ and very low abundances of $\mathrm{Zr}(34-60 \mathrm{ppm})$, $\mathrm{Sr}$ (47-94 ppm), Y (16-27 ppm), and $\mathrm{Nb}$ (0.5-1.2 ppm). Conversely, they contain higher abundances of $\mathrm{Al}_{2} \mathrm{O}_{3}$ $(14.2-18.0 \%)$ and $\mathrm{CaO}(11.3-13.4 \%)$ than most MORB,
Table 6. Preliminary results of 1-atm. melting experiments.

\begin{tabular}{|c|c|c|c|c|c|c|c|c|c|}
\hline \multirow[b]{2}{*}{ Run } & \multirow[b]{2}{*}{$\mathrm{T}\left({ }^{\circ} \mathrm{C}\right)$} & \multirow[b]{2}{*}{ Hours $^{\mathrm{a}}$} & \multicolumn{7}{|c|}{ Sample } \\
\hline & & & $504 \mathrm{~B}-1$ & 504B-9 & $504 \mathrm{~B}-11$ & $504 \mathrm{~B}-12$ & $504 \mathrm{~B}-21$ & 504B-53 & $501-16$ \\
\hline 11 & 1276 & 20.5 & - & - & - & - & $L^{b}$ & L & - \\
\hline 12 & 1265 & 23.5 & - & L. & - & L & L. & L. & L \\
\hline 5 & 1243 & 23.2 & L & LP & L & LP & - & - & LO \\
\hline 13 & 1237 & 20.0 & - & - & - & - & LP & L & - \\
\hline 10 & 1233 & 20.3 & L & LP & L & LP & - & - & LO \\
\hline 9 & 1227 & 24.5 & LP & LP & LP & LPO & - & - & LOP \\
\hline 14 & 1221 & 25.3 & - & LPO & - & - & LPO & L & - \\
\hline 4 & 1217 & 23.5 & LPO & LPOC & LPO & LPO & - & - & LOPC \\
\hline 16 & 1210 & 22.7 & - & - & - & - & LPO & L & - \\
\hline 7 & 1202 & 22.5 & LPOC & LPOC & LPO & LPO & - & - & LOPC \\
\hline 17 & 1193 & 26.3 & - & - & - & - & LPOC & - & - \\
\hline 2 & 1193 & 20.5 & LPOC & LPOC & LPOC & LPOC & - & - & LOPC \\
\hline 15 & 1186 & 66.7 & - & - & - & - & LPOC & - & - \\
\hline 8 & 1175 & 95.7 & LPOC & - & LPOC & LPOC & - & - & LOPC \\
\hline 1 & 1158 & 20.7 & LPOC & LPOC & POC & LPOC & - & - & LOPC \\
\hline 3 & 1144 & 99.5 & LPOC & LPOC & POC & POC & - & - & LOPC \\
\hline 6 & 1114 & 238.8 & LPOC & POC & POC & - & - & - & OPC \\
\hline
\end{tabular}

and as a consequence they have higher $\mathrm{CaO} / \mathrm{Na}_{2} \mathrm{O}$ ratios (5-8) than most ocean-floor basalts described to date (Melson et al., 1976). This in turn leads to higher than normal calculated values of anorthite in plagioclase $\left(\mathrm{An}_{78-84}\right)$ (Drake, 1976).

The Group D basalts, like the Group M basalts, are moderately evolved, with $\mathrm{Mg}^{\prime}$ values of 0.60 to 0.70 and abundances of $\mathrm{Cr}$ of 210 to $490 \mathrm{ppm}$, of Ni, 75 to 165 ppm, of $\mathrm{MgO}, 7.3$ to $9.0 \%$, and of $\mathrm{FeO}^{*}, 7.5$ to $10.5 \%$. However, the Group D basalts have higher liquidus temperatures $\left(1230-1265^{\circ} \mathrm{C}\right.$, Fig. 4) than the Group M basalts, and most appear to lie along the olivine-plagioclase cotectic, with some samples also saturated in clinopyroxene (Figs. 1 and 2; Walker et al., 1979).

Figure 5 compares the Costa Rica Rift zone basalts with Type I and II ocean-floor basalts (Bryan et al., 1976; Melson et al., 1976) with respect to $\mathrm{MgO}$ and FeO*. A few of the samples show the effects of olivine addition or slight alteration. A calculated olivine fractionation path $\left(\mathrm{K}_{\mathrm{D}}=0.3\right.$, fractionation increment $=$ 0.02 ) from a depleted, picritic parental melt (Elthon, 

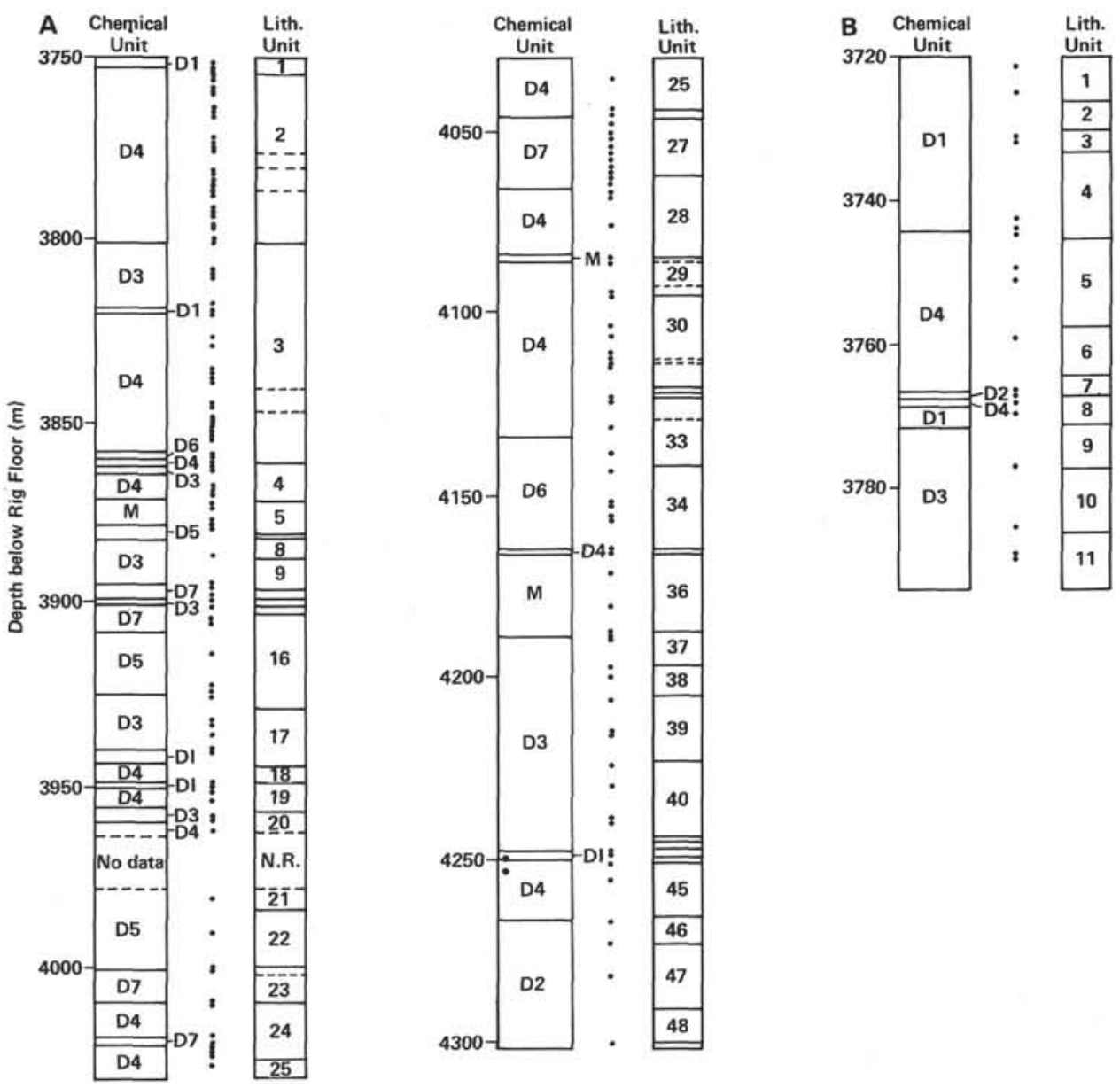

Figure 3. Stratigraphy for Holes 504B and 501. Small dots mark sample location and include those analyzed by Etoubleau et al. (this volume). Lithologic units are numbered as in the Site 501 and 504 chapters (this volume) and indicate separate flows, textures, or petrographic units. Chemical units are explained in the text. Note the approximate correspondence of lithologic and chemical units. N. R. indicates no recovery. (A) Hole 504B. (B) Hole 501.

1979 ) is also indicated in Figure 5. Figure 6, a plot of $\mathrm{Ni}$ versus $\mathrm{TiO}_{2}$, illustrates the depletion of the Costa Rica Rift zone samples in $\mathrm{TiO}_{2}$ with respect to "typical" MORB for their state of evolution. A possible fractionation path calculated for "normal" Type I basalts (olivine alone until $\mathrm{Ni}$ drops to $250 \mathrm{ppm}, 23 \%$ olivine plus $77 \%$ plagioclase for the $\mathrm{Ni}$ range from 250 to $50 \mathrm{ppm}$ ) is shown for reference. The basalts from this study must follow a similar path but at lower (depleted) $\mathrm{TiO}_{2}$ values. A plot of $\mathrm{Zr}$ versus Ni would lead to similar conclusions for the behavior of $\mathrm{Zr}$ in these samples.

The high degree of depletion in the Costa Rica Rift zone basalts is obvious from a plot of $\mathrm{TiO}_{2}$ versus $\mathrm{CaO}$ / $\mathrm{Na}_{2} \mathrm{O}$ (Fig. 7). The samples in this study exhibit very high $\mathrm{CaO} / \mathrm{Na}_{2} \mathrm{O}$ values in comparison to most oceanfloor basalts (the outlined field; Melson et al., 1976; Bryan et al., 1976). The primary picrite proposed by Elthon (1979) is shown again for reference. Only a few examples of similarly depleted ocean-floor basalts have been found previously: several rock samples from Indian Ocean (Melson et al., 1976), a glass inclusion in spinel (Donaldson and Brown, 1977), and a melt inclusion in olivine (Dungan and Rhodes, 1978). If these depleted magmas were mixed with magma of more normal composition, as might occur in shallow crustal magma chambers (O'Hara, 1977), the opportunity would exist for the generation of the enigmatic high anorthite disequilibrium phenocrysts commonly found in phyric ocean-floor basalts (Rhodes, Dungan, et al., 1979; Duncan and Green, 1980; Autio and Rhodes, 1981).

Both Groups $\mathrm{M}$ and $\mathrm{D}$ have $\mathrm{Zr} / \mathrm{Nb}$ ratios $\geq 40$ (Fig. 8 ), insofar as can be determined experimentally given the very low $\mathrm{Nb}$ value. These ratios are similar to those of the Type I ocean-floor basalts found on Legs 9, 34, and 45 but are in contrast to those of the Type II basalts found on Leg 49 and during the Franco-American MidOcean Undersea Study (FAMOUS) of the Mid-Atlantic Ridge at $45^{\circ} \mathrm{N}$, where the $\mathrm{Zr} / \mathrm{Nb}$ ratios are typically $\leq 10$ (Erlank and Kable, 1976; Rhodes, Blanchard, et al., 1979; Dungan, Rhodes, et al., 1979; Rhodes, Dungan, et al., 1979; Tarney et al., 1979; Cann et al., 1979; Basaltic Volcanism, 1981). Despite their similar $\mathrm{Nb} / \mathrm{Zr}$ ratios there is a large hiatus between the two Costa Rica Rift zone groups. 

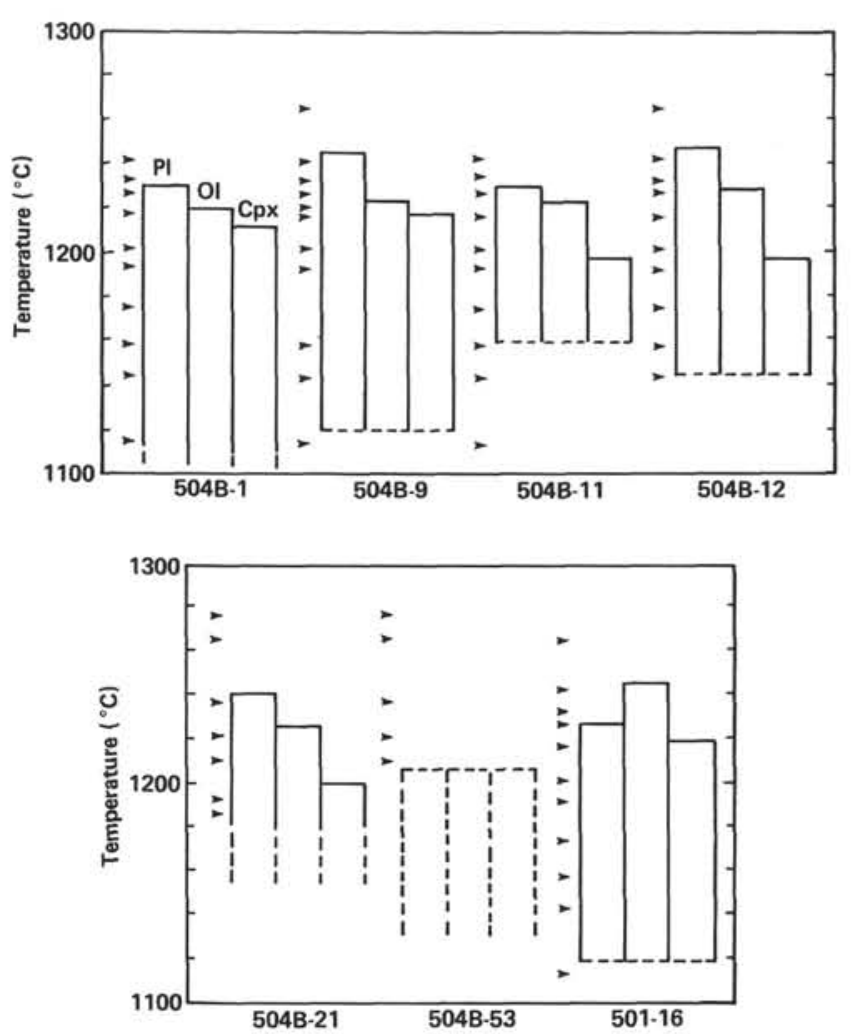

Figure 4. Preliminary 1-atm. experimental crystallization results for seven Costa Rica Rift zone basalts. Arrows to the left of each sample indicate experiment temperatures. Assemblages are \pm spinel. These basalts are close to or at clinopyroxene saturation.

A plot of $\mathrm{Zr}$ vs. $\mathrm{TiO}_{2}$ (Fig. 9) shows similar depletion of the Group D samples relative to most Type I and II basalts and the Group $M$ basalts. However, in contrast to the $\mathrm{Zr} / \mathrm{Nb}$ ratios, the Group $\mathrm{D}$ and $\mathrm{M}$ basalts differ greatly in their $\mathrm{Zr} / \mathrm{TiO}_{2}$ ratios, although some of the shipboard analyses (Etoubleau et al., this volume) occupy the intervening area. The depletion of the Group D basalts is also apparent in plots of $\mathrm{Zr}$ versus $\mathrm{Y}$ and $\mathrm{TiO}_{2}$ versus $\mathrm{Y}$ (Figs. 10 and 11).

Figure 12, a diagram that uses $\mathrm{Nb}, \mathrm{Zr}, \mathrm{Ti}, \mathrm{Y}$, and $\mathrm{V}$ as rare earth element analogs (Bougault, 1980), summarizes the important points of the preceding discussion: (1) a gap exists between Group M and Group D, especially at the "light" end of the diagram (where the magmaphile elements are most incompatible); (2) the two groups have similar $\mathrm{Zr} / \mathrm{Nb}$ ratios; and (3) the two groups have very different $\mathrm{Zr} / \mathrm{Ti}$ ratios.

\section{RARE EARTH ELEMENTS}

Three samples were selected for REE analysis by isotope dilution on the basis of XRF trace element data. The samples studied are Sample 504B-21, one of the most depleted samples (D7); Sample 504B-9, one of the moderately depleted samples (D4); and Sample 504B53, one of the Group M samples. REE abundances are given in Table 4 and plotted relative to chondritic abundances in Figure 13. Samples 504B-21 and 504B-9 have much lower light rare earth element (LREE) abundances than most MOR basalts (Basaltic Volcanism, 1981). The pattern for Sample 504B-9 is similar to that for 504B-21, but the REE abundances are slightly higher. The similarity of the abundances and patterns of these two depleted basalts to those of some komatiites (Whitford and Arndt, 1978; Echeverría, 1980) reemphasizes the high degree of depletion of the Costa Rica Rift zone basalts.

The REE abundances and pattern of Sample 504B-53 are different from those of the depleted basalts. This Group M sample exhibits an almost flat REE pattern and is enriched in all of the REE compared with the other two basalts. All three samples show slight positive (Sample 504B-21) or negative (Sample 504B-9, 504B-53) $\mathrm{Eu}$ anomalies. Five samples dredged from the modern rift and analyzed by Schilling et al. (1976) show very similar patterns and lie within the boundaries of Samples 504B-21 and 504B-53.

\section{STRATIGRAPHY}

As mentioned previously, diagrams of $\mathrm{TiO}_{2}$ versus $\mathrm{Zr}$ and $\mathrm{TiO}_{2}$ versus $\mathrm{Y}$ (Figs. 9 and 11) illustrate the similarity of the Group D basalts and the hiatus between the Group D and M basalts. The Group D samples were divided into seven subgroups (D1-D7) by using chemical criteria, D1 being the least depleted and D7 the most depleted. The premises used to separate the Costa Rica Rift zone basalts into chemical stratigraphic units were as follows:

1) Elements that were both relatively incompatible and resistant to alteration ( $\mathrm{Ti}, \mathrm{Zr}$, and $\mathrm{Y}$ ) were the best indicators of chemical units.

2) In juxtaposed samples, differences of less than $10 \%$ in these elements were probably the result of crystal fractionation.

3) In juxtaposed samples, differences of greater than $10 \%$ in these elements were probably the result of influxes of new magma batches.

Chemical stratigraphic columns for both Holes 504B and 501 were devised using both our own and the shipboard chemical analysis (Etoubleau et al., this volume). The resulting chemical stratigraphy corresponds closely to the shipboard-determined lithologic units (Fig. 3). The number of lithologic units may be larger than the number of units determined on the basis of the glass microprobe data (Natland et al., this volume) as a result of minor phenocryst addition and different criteria for unit changes. The cyclic nature of the magmatic processes is shown by the repetition and changing order of the subgroups in the stratigraphic columns (Fig. 3).

It should be emphasized that the analyses are much more similar within each chemical stratigraphic unit than between units (aside from minor variations due to crystal fractionation, alteration, and analysis precision). Table 5 gives the average composition and standard errors calculated for each of the eight subgroups found in the Hole 504B samples used in this study. A jump from Group D4 to D7, for example, represents a sizable change in chemical composition that cannot be explained solely by fractionation processes. Thus, the chemical stratigraphy is based on sharp breaks in chemistry between chemically related groups and probably indicates sequen- 


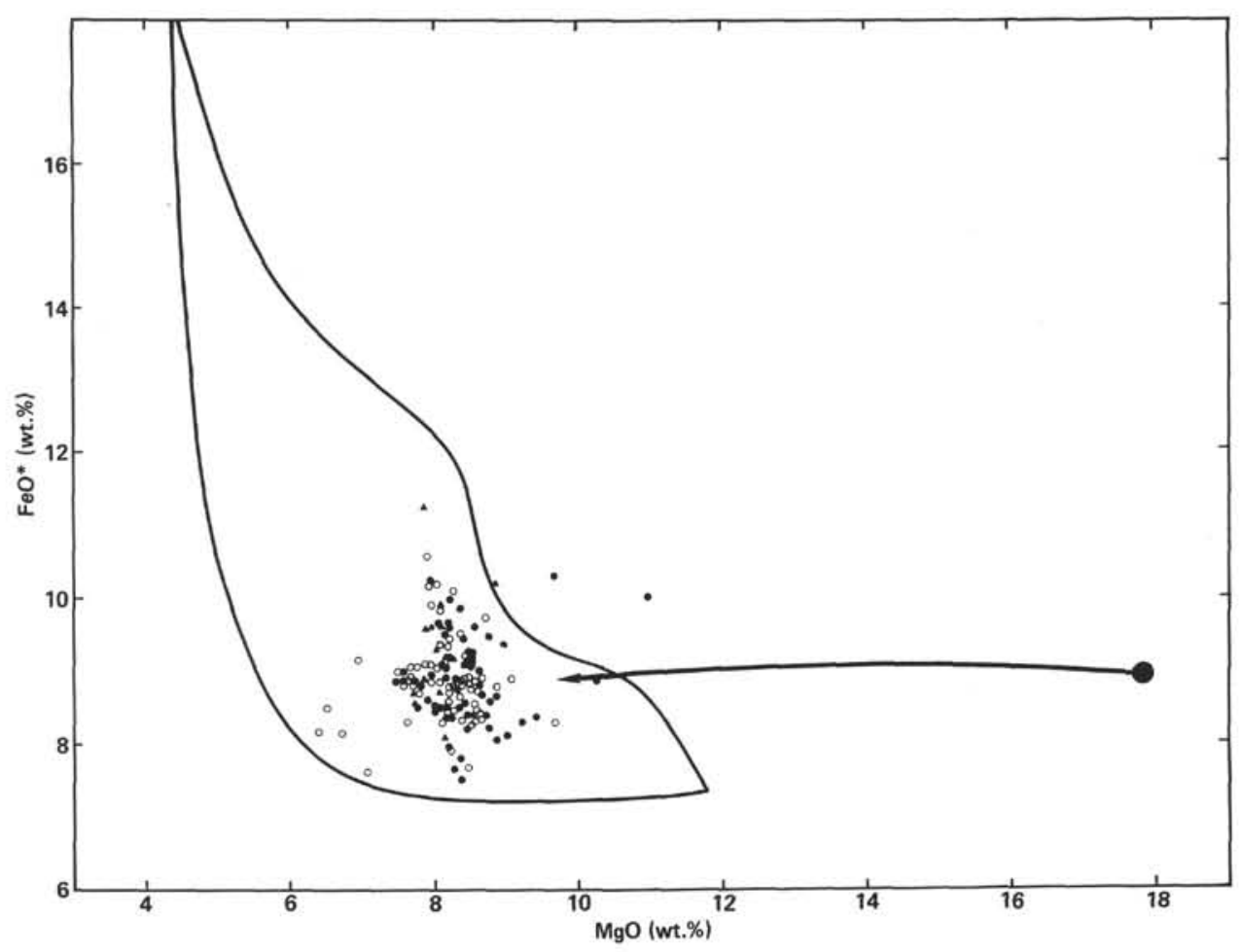

Figure 5. Plot of $\mathrm{MgO}$ versus $\mathrm{FeO}^{*}$, where $\mathrm{FeO}^{*}$ is total $\mathrm{Fe}$ expressed as $\mathrm{FeO}$. A calculated olivine fractionation path (see text) from a proposed primary picrite (large solid circle, Elthon, 1979) is indicated for reference. Costa Rica Rift zone basalts are moderately evolved and are similar in these major elements to "typical" ocean-floor basalts of similar states of evolution. Symbols are as in Figure 1, with open circles indicating the shipboard Hole 504B analyses (Etoubleau et al., this volume). A field encompassing typical MORB glass values is also shown (Melson et al., 1976).

tial influxes of magma into a differentiating magma chamber and/or separate flows from different magma chambers.

\section{EXPERIMENTAL PETROLOGY}

The initial results of the melting experiments are shown in Figure 4 and Table 6 . Preliminary microprobe data, which are consistent with calculated values of olivine and plagioclase (Roeder and Emslie, 1970; Drake, 1976), indicate an approach to equilibrium. The samples selected for the melting experiments represent a wide variety of chemical compositions and include one that is highly depleted (Sample 504B-21 [D7]); three that are moderately depleted (Samples 504B-9 [D4], 504B-11 [D3], and 501-16 [D3]); two that are less depleted (Samples 504B-1 [D1] and 504B-12 [D1]); and one that belongs to Group M (Sample 504B-53).

All of the depleted (Group D) samples exhibited the following crystallization sequence: small amounts of olivine or plagioclase, followed by olivine + plagioclase, followed by olivine + plagioclase + clinopyroxene. All assemblages are \pm spinel, which appears primarily as an artifact of small variations in the iron content of the sample holder wires. The small differences in the olivine and plagioclase liquidus temperatures $\left(5-20^{\circ} \mathrm{C}\right)$ indicate that these samples probably lie on or near the olivine-plagioclase cotectic. Slight phenocryst addition or mixing along a curved cotectic could also account for most of the differences. The appearance of clinopyroxene within $\sim 30^{\circ} \mathrm{C}$ of the liquidus is also evidence of the evolved nature of these basalts.

Although the chemical and experimental evidence indicates that all the Group D basalts are moderately evolved, they have anomalously high liquidus temperatures in comparison to basalts of similar degrees of evolution (i.e., those that have moderate $\mathrm{Mg}^{\prime}$ values and are near multiple saturation) (Dungan, Long, et al., 1978; Bender et al., 1978; and Walker et al., 1979). The liquidus temperatures calculated from major element compositions (French and Cameron, 1981) are approximately $30^{\circ} \mathrm{C}$ lower than the values measured for the samples. Depletion in the lower melting temperature magmaphile elements (particularly $\mathrm{Na}$ and $\mathrm{Ti}$ ) may result in raised liquidus temperatures of Group D. In keeping with "typical" MORB of similar composition, the relatively undepleted Group M sample, 504B-53, was still completely liquid at $1210^{\circ} \mathrm{C}$.

The liquidus temperatures of the three primary phases (olivine, plagioclase, and clinopyroxene) vary nonsystematically from sample to sample within the Group D basalts (Fig. 4). For example, clinopyroxene begins to crystallize at roughly $1220^{\circ} \mathrm{C}$ for Samples 504B-9 (D4) and 501-16 (D4), $1205^{\circ} \mathrm{C}$ for Samples 504B-1 (D1) and 504B-21 (D7), and $1195^{\circ} \mathrm{C}$ for Samples 504B-11 (D3) and 504B-12 (D1). The variation may result in part from slight phenocryst addition, but it also reflects the complexity of the evolution of these magmas. 


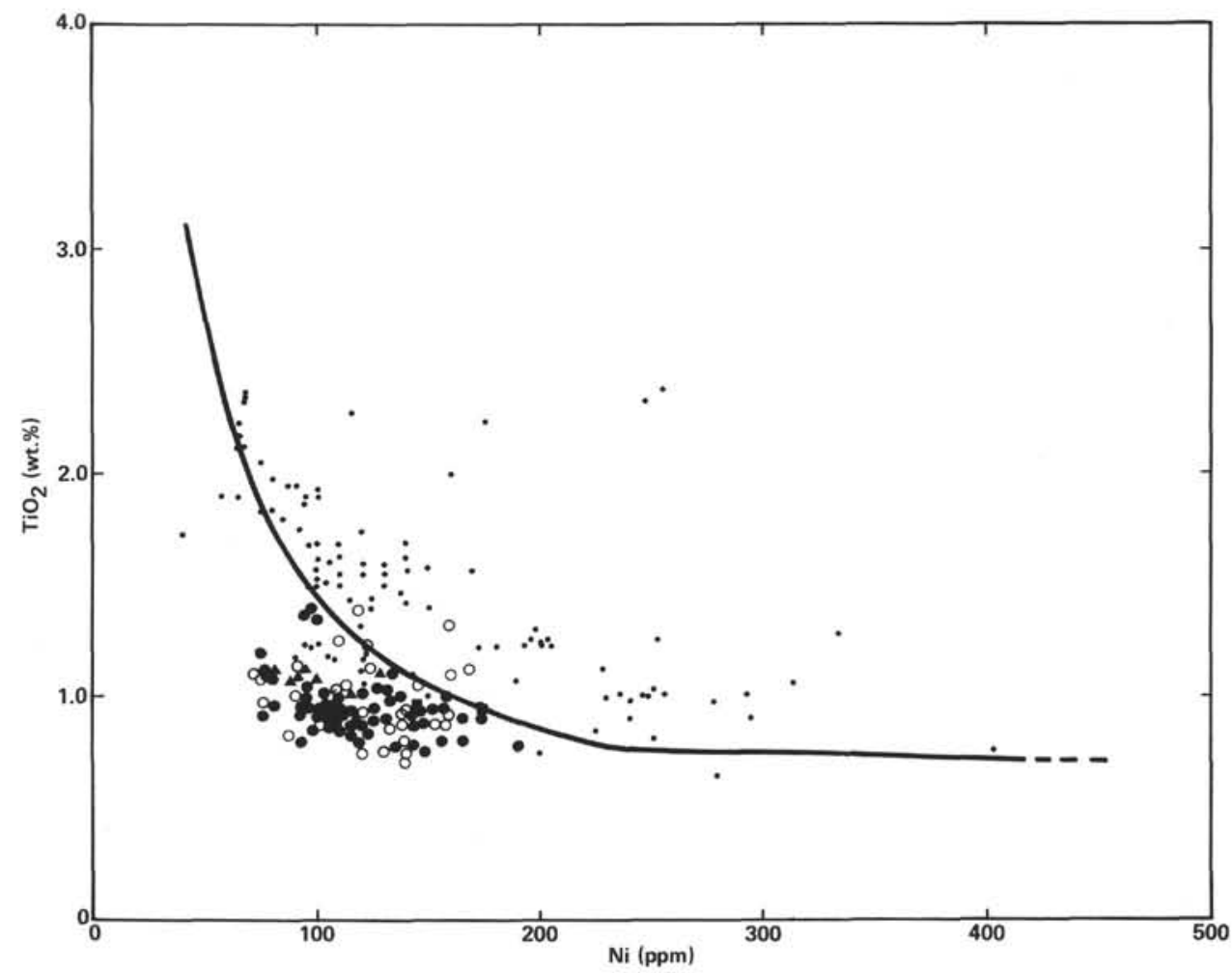

Figure 6. Plot of Ni versus $\mathrm{TiO}_{2}$. A calculated reference curve for the fractionation of "typical" MORB (see text) shows the depletion of the Costa Rica Rift zone basalts for their state of evolution. Symbols are as in Figure 5, with smaller dots indicating "typical" ocean-floor basalt glass analyses (Rhodes, 1973; Rhodes et al., 1976; Rhodes, Blanchard et al., 1979; and Tarney et al., 1979).

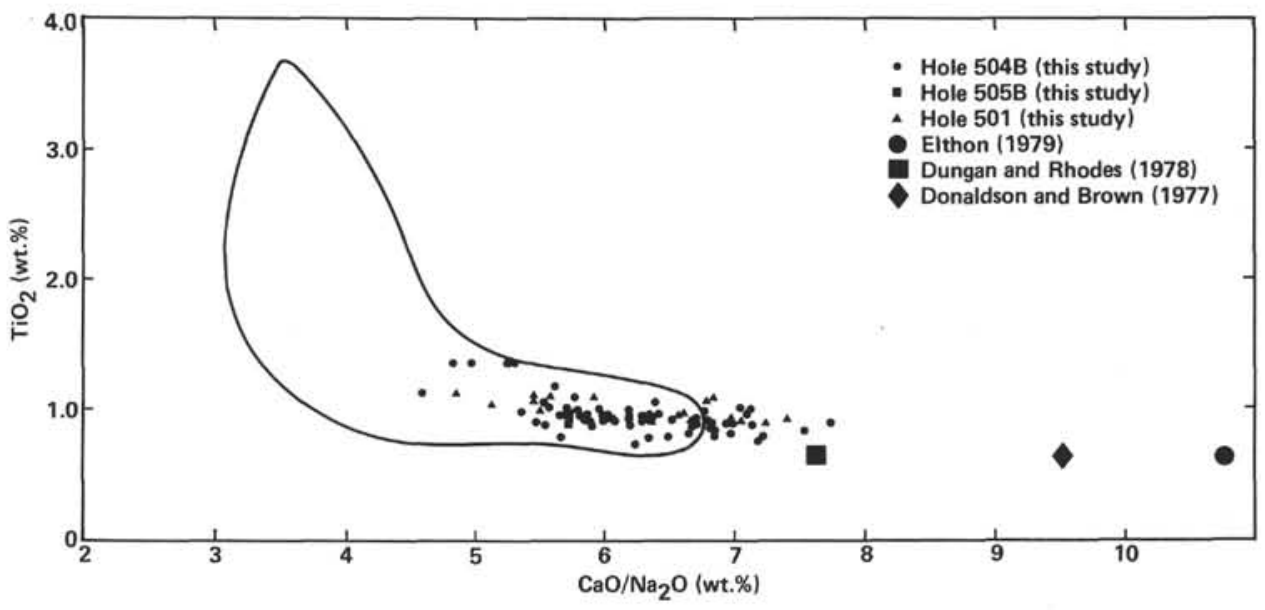

Figure 7. Plot of $\mathrm{CaO} / \mathrm{Na}_{2} \mathrm{O}$ versus $\mathrm{TiO}_{2}$. Note the high $\mathrm{CaO} / \mathrm{Na}_{2} \mathrm{O}$ ratios for the Costa Rica Rift zone basalts compared to the field shown for "normal" MORB glasses (Melson et al., 1976).

\section{DISCUSSION}

Several factors indicate that the Group D and Group $\mathrm{M}$ basalts are not comagmatic. The similarity of the $\mathrm{Mg}^{\prime}$ values (Tables 1 to 3 and 5) in combination with large differences in the abundances and ratios of incompatible elements support the conclusion that Groups M and $\mathrm{D}$ cannot be the result of the differentiation (through crystal fractionation, magma mixing, etc.) of a common parental magma. The trace, rare earth, and major ele- ment chemistry are also consistent with this conclusion (Figs. 6, 7, and 9-12). The lack of complete correlation between chemical assessment of depletion and liquidus temperature (i.e., the similarity of the liquidus temperatures of rocks judged to be depleted to varying degrees [Fig. 4]) is another indication that these rocks are not related by simple fractionation to a common parental magma. Although the Group D basalts appear to form a single differentiation sequence, they also reflect the influence of separate batches of magma of similar compo- 


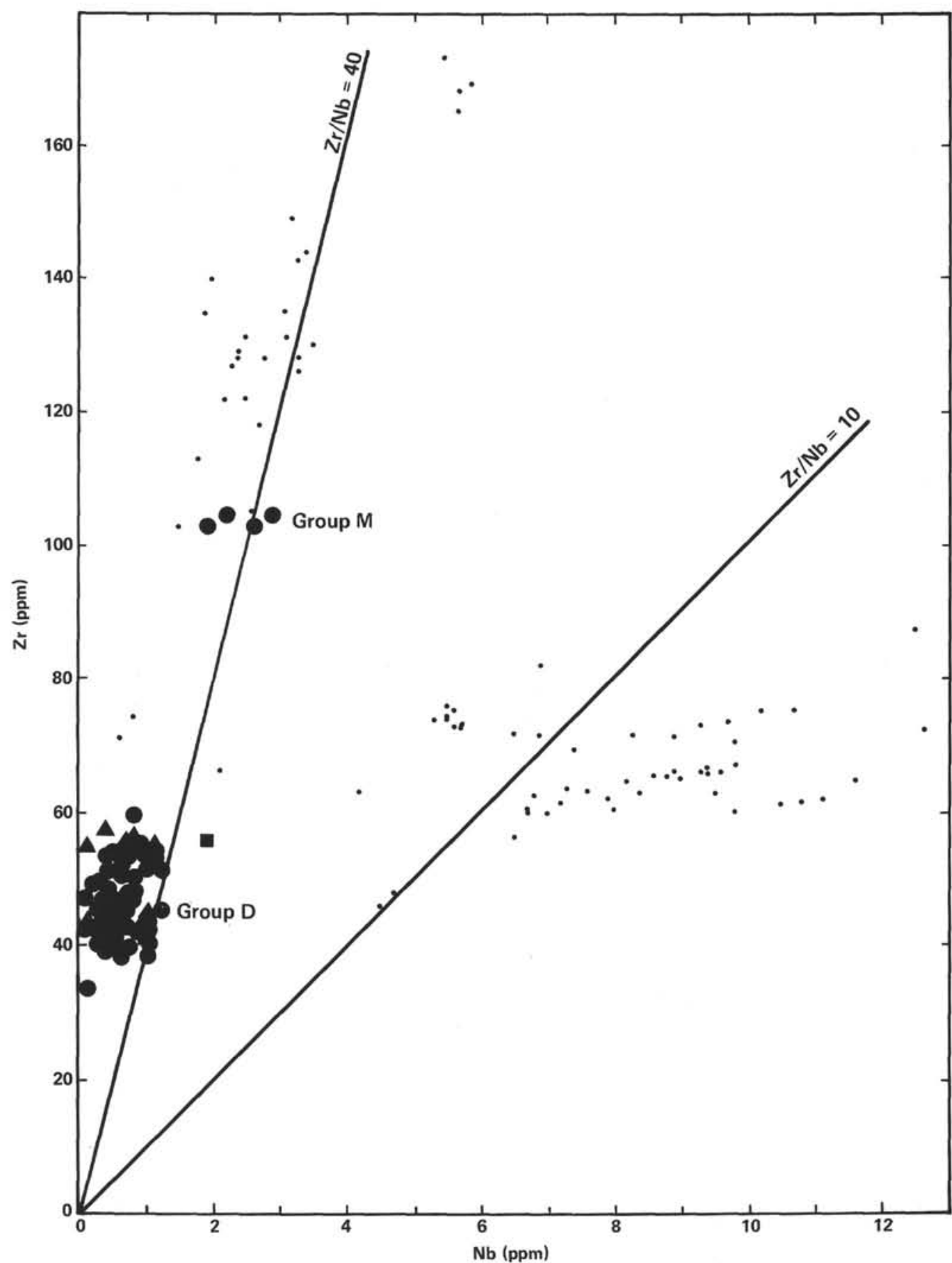

Figure 8. Plot of $\mathrm{Zr}$ versus $\mathrm{Nb}$. Costa Rica Rift zone basalts have $\mathrm{Zr} / \mathrm{Nb}$ ratios similar to Type I (Bryan et al., $1976)$ basalts $(\geq 40)$ and are unlike the more transitional Type II basalts $(\leq 10)$. Group D basalts have a much lower $(\sim 50 \%)$ abundance of $\mathrm{Zr}$ and $\mathrm{Nb}$ relative to Group M basalts and "normal" MORB. Symbols are as in Figure 1, with smaller dots representing "typical" ocean-floor basalt glass analyses (Rhodes, 1973; and Rhodes et al., 1976).

sition. Evidence for this includes the cited experimental inconsistencies and the interlayering of repeated magma types (Fig. 3).

The traditional explanation for major differences in magmaphile element abundances and LREE/HREE, $\mathrm{Zr}$ / $\mathrm{Ti}$, and $\mathrm{Zr} / \mathrm{Y}$ ratios has been two distinct mantle sources (Schilling, 1971). However, multistage or incremental melting of the mantle can produce similar changes between magma batches (Langmuir et al., 1977; Wood,
1979; Duncan and Green, 1980). Duncan and Green (1980) have proposed that two types of magma may form through multistage melting of the mantle. The first product is a more abundant tholeiitic picrite, which fractionates olivine to produce a melt similar to the most primitive ocean-floor basalt described to date (Frey et al., 1974). The refractory residue is remelted at shallower depth or higher temperature to produce a magma with lower LREE and magmaphile element abundances. This 


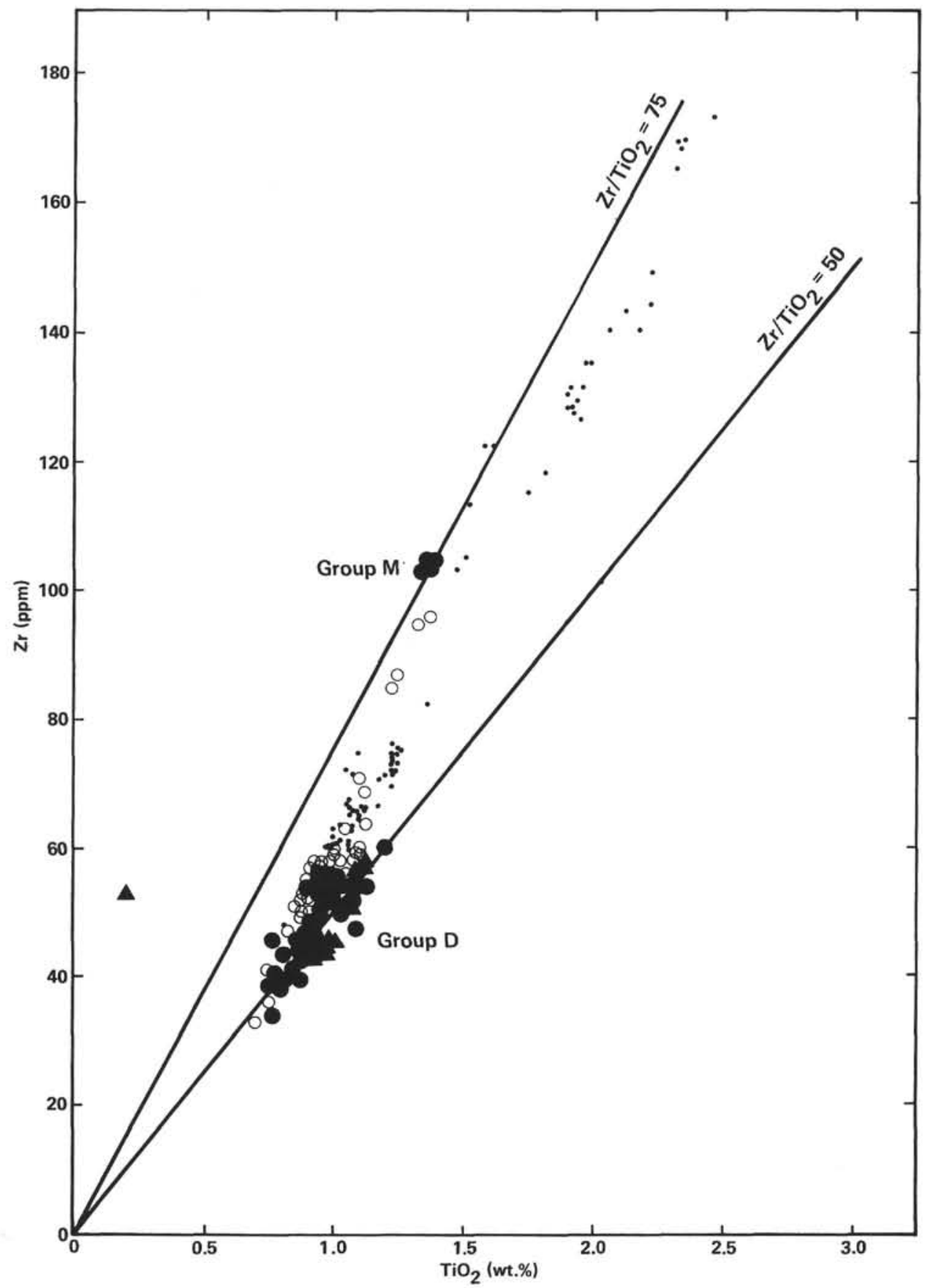

Figure 9. Plot of $\mathrm{Zr}$ versus $\mathrm{TiO}_{2}$. Group $\mathrm{D}$ basalts have lower $\mathrm{Zr} / \mathrm{TiO}_{2}$ ratios and lower $\mathrm{Zr}$ and $\mathrm{TiO}_{2}$ abundances than Group $\mathrm{M}$ basalts. A few shipboard samples (open circles, Etoubleau et al., this volume) fall between the two groups (see text). Symbols are as in Figure 8. MORB glass analyses are from Rhodes (1973) and Rhodes et al. (1976).

magma leaves a highly refractory depleted residue such as the lherzolite found at the Troodos Massif in Cyprus (Gass, 1968; Moores and Vine, 1971). Calculations based on Drake (1976) and Roeder and Emslie (1970) indicate that this second proposed melt is in equilibrium with the highly refractory phenocrysts of calcic plagioclase $\left(\mathrm{An}_{82-88}\right)$ and magnesian olivine $\left(\mathrm{Fo}_{90}\right)$ found in many phyric ocean-floor basalts.

Evidence for the existence of this second group of depleted magmas, which is largely indirect, is derived from experiments with natural systems (Jaques and Green, 1980) and studies of refractory phenocrysts and melt inclusions (Donaldson and Brown, 1977; Dungan and Rhodes, 1978). Descriptions of examples of ocean-floor basalts with these characteristics are rare, however, and include just a few glass analyses from the Indian Ocean (Melson et al., 1976).

As noted earlier, the Group $M$ basalts have the same chemical characteristics as "normal" moderately evolved Type I basalts. Consequently, the parental and possibly 


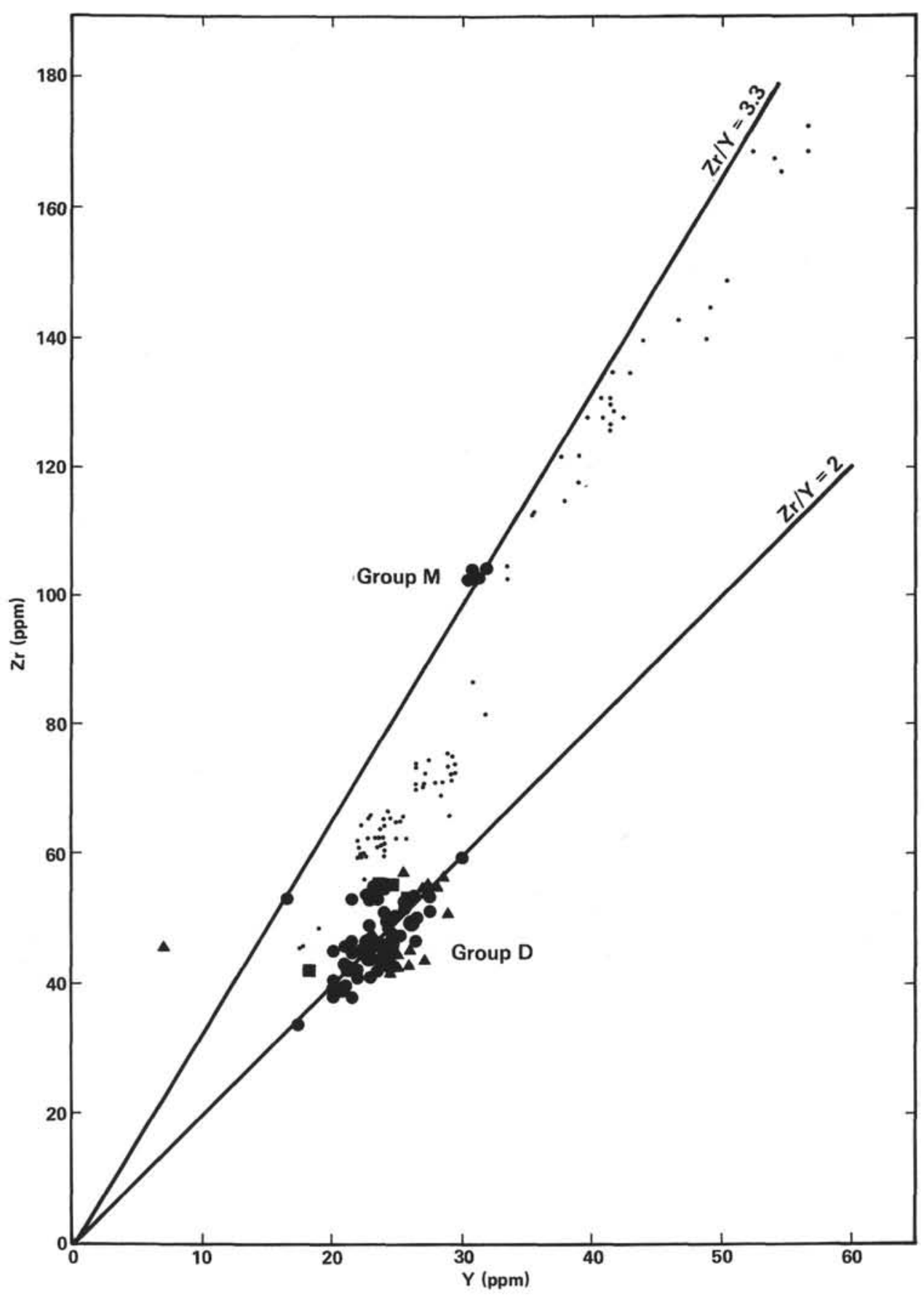

Figure 10. Plot of $\mathrm{Zr}$ versus $\mathrm{Y}$. Group D basalts have lower $\mathrm{Zr}$ and $\mathrm{Y}$ abundances than Group $\mathrm{M}$ and more "typical" ocean-floor basalts. They also have a much lower $\mathrm{Zr} / \mathrm{Y}$ ratio. Symbols are the same as in Figure 8. MORB glass analyses are from Rhodes (1973) and Rhodes et al. (1976).

primary magmas from which these basalts have evolved could be similar to the most primitive ocean-floor basalts described to date (Frey et al., 1974). The Group D basalts, on the other hand, are quite distinct from common ocean-floor basalts, particularly with respect to their $\mathrm{CaO} / \mathrm{Na}_{2} \mathrm{O}$ ratios (Fig. 7) and magmaphile element abundances. They are similar in some respects to the nearby Gorgona complex (Echeverría, 1980), which consists of interlayered komatiites and basalts and is also depleted in LREE and incompatible elements.
Figures 5 and 7 show that Group D basalts could have differentiated from picritic melts similar to Elthon's (1979) proposed primary ocean-floor magma. Therefore, although most of the Costa Rica Rift zone basalts are depleted in the magmaphile elements and have LREE abundances and $\mathrm{CaO} / \mathrm{Na}_{2} \mathrm{O}$ ratios more similar to komatiites and picrites, the $\mathrm{MgO}$ versus $\mathrm{FeO}^{*}$ diagram (Fig. 5) shows that all of these samples are much more evolved than picrites (and, indeed, are more evolved than the most primitive ocean-floor basalts described to 


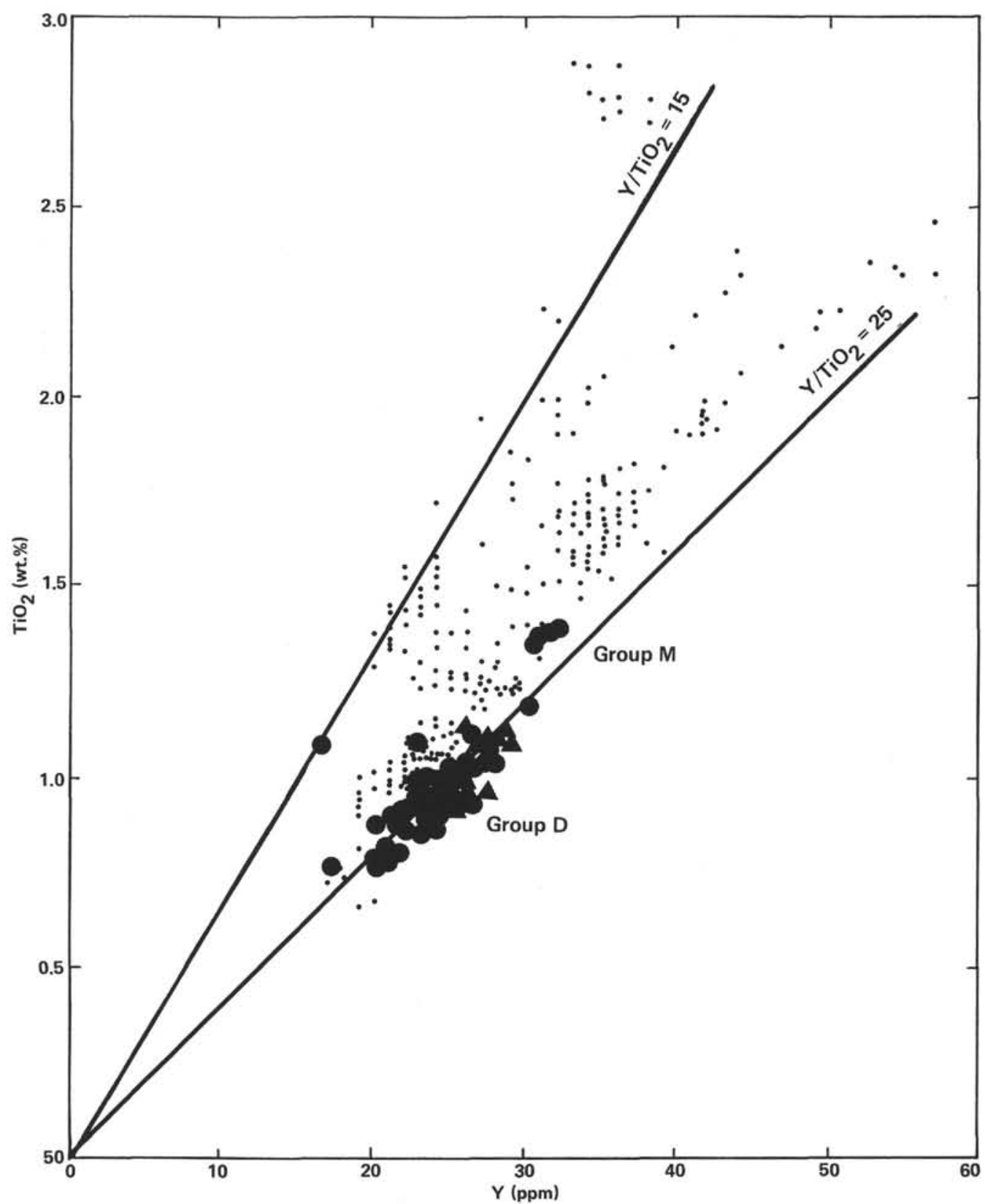

Figure 11. Plot of $\mathrm{TiO}_{2}$ versus $\mathrm{Y}$. Group $\mathrm{M}$ and $\mathrm{D}$ basalts have similar $\mathrm{Y} / \mathrm{TiO}_{2}$ ratios. All of the Costa Rica Rift Zone basalts have low $\mathrm{TiO}_{2}$ and $\mathrm{Y}$ abundances compared to "typical" MORB. Symbols are the same as in Figure 8. MORB glass analyses are from Rhodes (1973) and Rhodes et al. (1976).

date; Frey et al., 1974). Thus, these samples could be produced from picrites only by extensive crystal fractionation.

The stratigraphic interlayering of the Group $\mathrm{M}$ and $\mathrm{D}$ basalts, coupled with essentially identical ratios of highly incompatible elements (such as $\mathrm{Zr} / \mathrm{Nb}$; see Fig. 12) is strong evidence that the two major basalt types seen at the Costa Rica Rift zone did not have separate sources. If correct, the observed variations in LREE/HREE, $\mathrm{Zr}$ / $\mathrm{Y}$, and $\mathrm{Zr} / \mathrm{Ti}$ ratios and the large differences in magmaphile element abundances suggest that incremental or multistage melting of a single source generated the precursors of both the Group M and D basalts. According to this model, two types of basalt were produced in this way: an early, less depleted basalt similar to the primitive Type I basalts (Bryan et al., 1976) that evolved into the Group $M$ basalts and a larger fraction of later basalts more depleted in LREE and magmaphile elements that evolved into the Group D basalts. This model requires that clinopyroxene remain in the source for both melts for the element ratios and abundances to work properly.

The dominance of depleted magmas in the Costa Rica Rift zone may mean that there was not enough "typical" MORB magma in the first stage to swamp the chemical signature of the later melts (Group D) during mixing in shallow magma chambers. Rather, only an occasional pod of early melt (Group M) made it to the sur- 


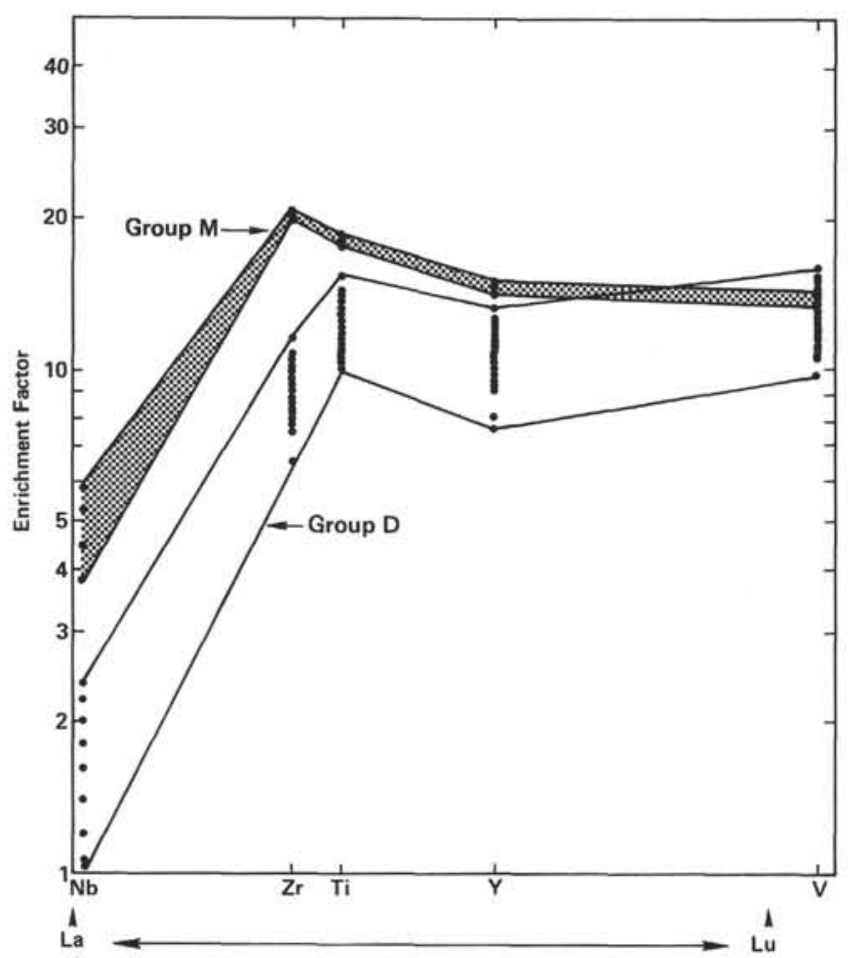

Figure 12. Plot using normalized trace elements $(\mathrm{Nb}, \mathrm{Zr}, \mathrm{Ti}, \mathrm{Y}, \mathrm{V})$ as REE analogs (Bougault, 1980). Both Group M and D basalts are depleted in LREE analogs. Group M is not continuous with Group $\mathrm{D}$ and exhibits a different $\mathrm{Zr} / \mathrm{Ti}$ ratio but a similar $\mathrm{Zr} / \mathrm{Nb}$ ratio. Small dots indicate individual analyses. Group $M$ field is shaded.

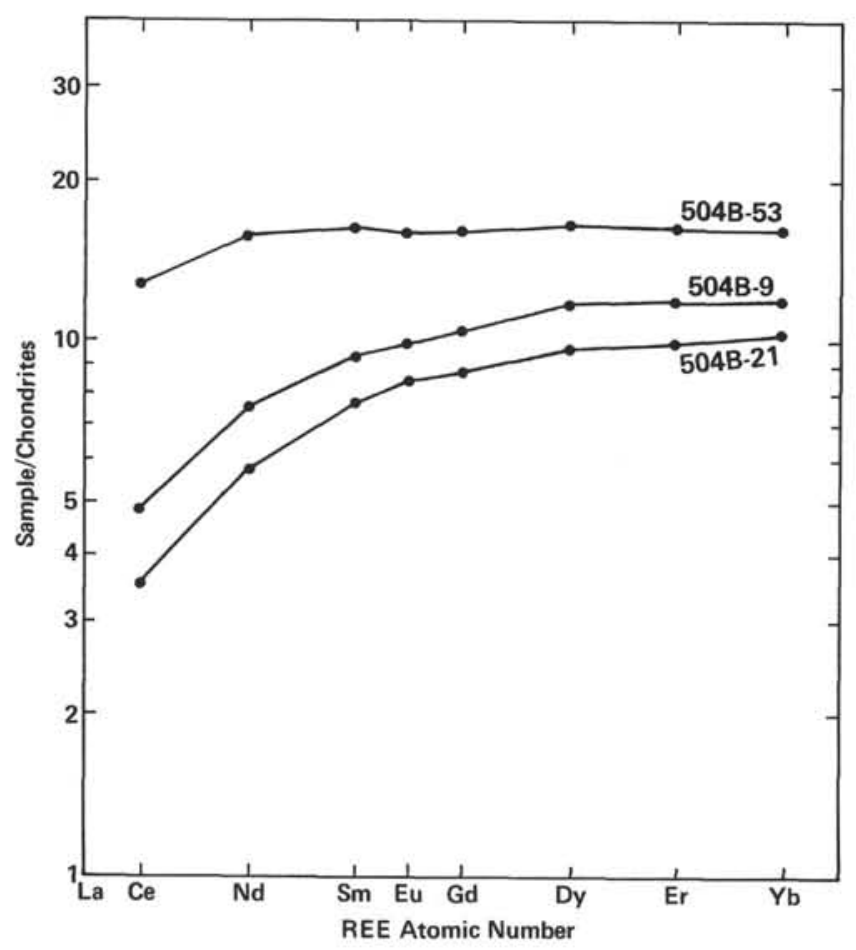

Figure 13. Plot of normalized REE abundances for three Costa Rica Rift zone basalts. Notice the LREE and REE depletion of the Group D basalts (Samples 504B-9 and 504B-21) relative to the Group M basalt (Sample 505-53). However, all of these samples are LREE depleted. Analyses were performed by J. Bender. face or mixed with later magmas. The variety of noncomagmatic or derivative magma types seen here may indicate that no extensive magma chamber exists along the length of the Costa Rica Rift as is proposed for the East Pacific Rise (Rosendahl, 1976). Rather, we suggest a series of smaller, nearly steady-state magma chambers similar to those proposed for the Mid-Atlantic Ridge (Rosendahl, 1976; Bryan and Moore, 1977). The chambers would not be small enough to solidify to any great extent between eruptions (they fail to produce FeTi basalts); and the repetition of magma types suggests a steady-state process (O'Hara, 1977; O'Hara and Mathews, 1981). The recycling of previously melted mantle (from the East Pacific Rise) might explain the lack of volumetrically significant "typical" MORB.

Even the most primitive ocean-floor basalt compositions described to date (Frey et al., 1974) fail to produce the very calcic disequilibrium plagioclase phenocrysts so common in phyric MORB samples (Bryan et al., 1976; Rhodes, Blanchard, et al., 1979; Dungan and Rhodes, 1978; Duncan and Green, 1980). However, the composition of the Costa Rica Rift zone Group D basalts approaches that needed to produce such phenocrysts. Mixing small amounts of magmas like these with more "typical" MORB magmas in shallow magma chambers would provide a source for these phenocrysts in the phyric ocean-floor basalts without drastically changing the chemistry of the more voluminous "typical" MORB. If depleted magmas similar to those found at the Costa Rica Rift zone are the source of the calcic plagioclase phenocrysts, then depleted magmas must be a small but pervasive component of the ocean-floor basalt system.

\section{CONCLUSIONS}

1. There are two major basalt groups in the Costa Rica Rift zone samples, one similar to "typical" MORB (Group M) and one highly depleted in magmaphile elements (Group D).

2. Both groups are moderately evolved, with both olivine and plagioclase and often clinopyroxene on the liquidus. However, the liquidus temperatures of Group $\mathrm{D}$ are higher, possibly because of their depletion in the magmaphile elements with lower melting temperatures.

3. Group $\mathrm{D}$ basalts have high $\mathrm{CaO} / \mathrm{Na}_{2} \mathrm{O}$ ratios and are calculated to be in equilibrium with highly anorthitic plagioclase $\left(\mathrm{An}_{78-84}\right)$. When basalts like these are mixed with normal MORB magmas in shallow magma chambers they may provide a source for the highly calcic disequilibrium phenocrysts often found in "typical" MORB samples.

4. Cyclical chemical stratigraphy indicates influxes of new magma into a differentiating magma chamber or separate flows from different magma chambers or both.

5. Constant $\mathrm{Zr} / \mathrm{Nb}$ ratios suggest a single mantle source for the two groups, with major differences (such as those in $\mathrm{Zr} / \mathrm{Ti}$ and LREE/HREE) arising through multiple melting episodes, with lesser effects from magma mixing and fractional crystallization. Thus, Group $\mathrm{M}$ may have arisen through fractionation of a primitive MORBlike first melting product, and Group D may be the result of fractionation from a possibly picritic and 
certainly depleted product from a later melting episode of the same source.

\section{ACKNOWLEDGMENTS}

J. Natland kindly provided the rock samples for this study. We wish also to thank J. Bender for his careful isotope dilution determinations and D. Walker for the generous use of his experimental laboratory. M. Dungan, J. Bender, and J. Natland gave perceptive reviews which greatly improved the organization of this paper. We particularly wish to thank J. Sparks for technical assistance. This study was supported by two NSF grants to J. M. Rhodes: OCE78-26330 and EAR78-19892.

\section{REFERENCES}

Autio, L. K., and Rhodes, J. M., 1981. Basalts from Holes 501 and 504B (Costa Rican Rift Zone): A possible source for high anorthite phenocrysts in ocean-floor basaltic magmas. Eos, 62:309.

Basaltic Volcanism on the Terrestrial Planets, 1981: New York (Pergamon Press, Inc.).

Bender, J. F., Hodges, F. N., and Bence, A. E., 1978. Petrogenesis of basalts from the project FAMOUS area: Experimental study from 0 to 15 kbars. Earth Planet. Sci. Lett., 41:277-302.

Bernas, B., 1968. A new method for decomposition and comprehensive analysis of silicates by atomic absorption spectrometry. Anal. Chem., 40:1682-1686.

Bougault, H., 1980. Contribution des éléments de transition à la comprehension de la genèse des basaltes oceaniques [Ph.D. dissert.]. Université de Paris VII, France.

Bryan, W. B., and Moore, J. G., 1977. Compositional variations of young basalts in the Mid-Atlantic Ridge rift valley near lat $36^{\circ} 49^{\prime}$ N. Geol. Soc. Am. Bull., 88:556-570.

Bryan, W. B., Thompson, G., Frey, F. A., and Dickey, J. S., 1976. Inferred geological settings and differentiation in basalts from the Deep-Sea Drilling Project. J. Geophys. Res., 81:4285-4304.

Cann, J. R., Tarney, J., Varet, J., and Wood, D. A., 1979. Mantle heterogeneity in the North Atlantic: evidence from Leg 49 geochemistry. In Luyendyk, B. P., Cann, J. R., et al., Init. Repts. DSDP, 49: Washington (U.S. Govt. Printing Office), 841-850.

Donaldson, C. H., and Brown, R. W., 1977. Refractory megacrysts and magnesium-rich melt inclusions within spinel in oceanic tholeiites: Indicators of magma mixing and parental magma composition. Earth Planet. Sci. Lett., 37:81-89.

Drake, M. J., 1976. Plagioclase-melt equilibria. Geochim. Cosmochim. Acta, 40:457-466.

Duncan, R. A., and Green, D. H., 1980. Role of multistage melting in the formation of oceanic crust. Geology, 8:22-26.

Dungan, M. A., Long, P. E., and Rhodes, J. M., 1979. The petrology, mineral chemistry, and one-atmosphere phase relations of basalts from Site 395. In Melson, W. G., Rabinowitz, P. D., et al., Init. Repts. DSDP, 45: Washington (U.S. Govt. Printing Office), 461-478.

Dungan, M. A., and Rhodes, J. M., 1978. Residual glasses and melt inclusions in basalts from DSDP Legs 45 and 46: Evidence for magma mixing. Contrib. Mineral. Petrol., 67:417-431.

Dungan, M. A., Rhodes, J. M., Long, P. E., Blanchard, D. P., Brannon, J. C., and Rodgers, K. V., 1979. Petrology and geochemistry of basalts from Site 396, Legs 45 and 46 of the Deep Sea Drilling Project. In Dmitriev, L., Heirtzler, J., et al., Init. Repts. DSDP, 46: Washington (U.S. Govt. Printing Office), 89-113.

Echeverría, L. M., 1980. Tertiary or Mesozoic komatiites from Gorgona Island, Columbia: Field relations and geochemistry. Contrib. Mineral. Petrol., 73:253-266.

Elthon, D., 1979. High magnesia liquids as the parental magma for ocean floor basalts. Nature, 278:514-518.

Erlank, A. J., and Kable, E. J. D., 1976. The significance of incompatible elements in Mid-Atlantic Ridge basalts from $45^{\circ} \mathrm{N}$ with particular reference to $\mathrm{Zr} / \mathrm{Nb}$. Contrib. Mineral. Petrol., 54: 281-291.

French, W. J., and Cameron, E. P., 1981. Calculation of the temperature of crystallization of silicates from basaltic melts. Mineral. Mag., 44:19-26.
Frey, F. A., Bryan, W. B., and Thompson, G., 1974. Atlantic Ocean floor: Geochemistry and petrology of basalts from Legs 2 and 3 of the Deep-Sea Drilling Project. J. Geophys. Res., 79:5507-5527.

Gass, I. G., 1968. Is the Troodos Massif of Cyprus a fragment of Mesozoic ocean floor? Nature, 220:39-42.

Hanson, G. N., 1976. Rare earth element analysis by isotope dilution. Natl. Bur. Stand. Spec. Publ., 422:937-949.

Jaques, A. L., and Green, D. H., 1980. Anhydrous melting of peridotite at $0-15 \mathrm{~Kb}$ pressure and the genesis of tholeiitic basalts. Contrib. Mineral. Petrol., 73:287-310.

Langmuir, C. H., Bender, J. F., Bence, A. E., and Hanson, G. N., 1977. Petrogenesis of basalts from the FAMOUS area: Mid-Atlantic Ridge. Earth Planet. Sci. Lett., 36:133-156.

Masuda, A., Nakamura, N., and Tanaka, T., 1973. Fine structures of mutually normalized rare-earth patterns of chondrites. Geochim. Cosmochim. Acta, 37:239-248.

Melson, W. G., Vallier, T. L., Wright, T. L., Byerly, G., and Nelen, J., 1976. Chemical diversity of abyssal volcanic glass erupted along Pacific, Atlantic, and Indian Ocean sea-floor spreading centers. In Sutton, G. H., Manghnani, M. H., and Moberly, R. (Eds.), The Geophysics of the Pacific Ocean Basin and its Margin: Washington, D.C., (Am. Geophys. Union), pp. 351-367.

Moores, E. M., and Vine, F. J., 1971. The Troodos Massif, Cyprus and other ophiolites as oceanic crust: evaluation and implications. Phil. Trans. Roy. Soc. Lond. A., 268:443-446.

Norrish, K., and Chappell, B. W., 1967. X-Ray fluorescence spectrometry. In Zussman, J., (Ed.), Physical Methods in Determinative Mineralogy: New York (Academic Press), pp. 161-214.

Norrish, K., and Hutton, J. T., 1969. An accurate X-ray spectrographic method for the analysis of a wide range of geological samples. Geochim. Cosmochim. Acta, 33:431-453.

O'Hara, M. J., 1968. Are ocean floor basalts primary magma? Nature, 220:683-686.

1977. Geochemical evolution during fractional crystallization of a periodically refilled magma chamber. Nature, 266: 503-507.

O'Hara, M. J., and Mathews, R. E., 1981. Geochemical evolution in an advancing, periodically replenished, periodically tapped, continuously fractionated magma chamber. J. Geol. Soc. London, $38: 237-277$.

Reynolds, R. C., 1967. Matrix corrections in trace element analysis by $X$-Ray Fluorescence: Estimation of the mass absorption coefficient by Compton scattering. Am. Mineral., 48:1133-1143.

Rhodes, J. M., 1973. Major and trace element chemistry of basalts from Leg 9 of the Deep Sea Drilling Project. Eos, 54:1014-1015.

Rhodes, J. M., Blanchard, D. P., Dungan, M. A., Rodgers, K. V., and Brannon, J. C., 1979. Chemistry of Leg 45 basalts. In Melson, W. G., Rabinowitz, P. D., et al., Init. Repts. DSDP, 45: Washington (U.S. Govt. Printing Office), 447-459.

Rhodes, J. M., Blanchard, D. P., Rodgers, K. V., Jacobs, J. W., and Brannon, J. C., 1976. Petrology and chemistry of basalts from the Nazca Plate: Part 2-Major and trace element chemistry. In Yeats, R. S., Hart, S. R. et al., Init. Repts. DSDP, 34: Washington (U.S. Govt. Printing Office), 239-244.

Rhodes, J. M., Dungan, M. A., Blanchard, D. P., and Long, P. E., 1979. Magma mixing at mid-ocean ridges: Evidence from basalts drilled near $22^{\circ} \mathrm{N}$ on the mid-Atlantic ridge. Tectonophysics, 55: $35-61$.

Roeder, P. L., and Emslie, R. F., 1970. Olivine-liquid equilibrium. Contrib. Mineral. Petrol., 29:275-289.

Rosendahl, B. R., 1976. Evolution of oceanic crust. 2. constraints, implications, and inferences. J. Geophys. Res., 81:5305-5314.

Schilling, J.-G., 1971. Sea-floor evolution: Rare-earth evidence. Phil. Trans. Roy. Soc. Lond. A., 268:663-706.

Schilling, J.-G., Anderson, R. N., and Vogt, P., 1976. Rare earth, Fe and $\mathrm{Ti}$ variations along the Galapagos spreading centre, and their relationship to the Galapagos mantle plume. Nature, 261:108-113.

Shido, F., Miyashiro, A., and Ewing, M., 1971. Crystallization of abyssal tholeiites. Contrib. Mineral. Petrol., 31:251-266.

Tarney, J., Saunders, A. D., Weaver, S. D., Donnellan, N. C. B., and Hendry, G. L., 1979. Minor-element geochemistry of basalts from Leg 49, North Atlantic Ocean. In Luyendyk, B. P., Cann, J. R., et 
al., Init. Repts. DSDP, 49: Washington (U.S. Govt. Printing Office), 657-691.

Walker, D., Shibata, T., and DeLong, S. E., 1979. Abyssal tholeiites from the oceanographer fracture zone. II. Phase equilibria and mixing. Contrib. Mineral. Petrol., 70:111-125.
Whitford, D. J., and Arndt, N. T., 1978. Rare earth element abundances in a thick, layered komatiite lava flow from Ontario, Canada. Earth Planet. Sci. Lett., 41:188-196.

Wood, D. A., 1979. Dynamic partial melting: Its application to the petrogeneses of basalts erupted in Iceland, the Faeroe Islands, the Isle of Skye (Scotland) and the Troodos Massif (Cyprus). Geochim. Cosmochim. Acta, 43:1031-1046. 